\title{
Electric dipole moments in a leptoquark scenario for the $B$-physics anomalies
}

\author{
Wolfgang Altmannshofer, ${ }^{a}$ Stefania Gori, ${ }^{a}$ Hiren H. Patel, ${ }^{a}$ Stefano Profumo ${ }^{a}$ \\ and Douglas Tuckler ${ }^{a, b}$ \\ ${ }^{a}$ University of California, Santa Cruz, and Santa Cruz Institute for Particle Physics, \\ Santa Cruz, CA 95064, U.S.A. \\ ${ }^{b}$ Fermi National Accelerator Laboratory, \\ Batavia, IL, 60510, U.S.A. \\ E-mail: waltmann@ucsc.edu, sgori@ucsc.edu, hpatel6@ucsc.edu, \\ profumo@ucsc.edu, dtuckler@ucsc.edu
}

ABSTRACT: Vector leptoquarks can address the lepton flavor universality anomalies in decays associated with the $b \rightarrow c \ell \nu$ and $b \rightarrow s \ell l$ transitions, as observed in recent years. While not required to explain the anomalies, these leptoquarks generically yield new sources of $\mathrm{CP}$ violation. In this paper, we explore constraints and discovery potential for electric dipole moments (EDMs) in leptonic and hadronic systems. We provide the most generic expressions for dipole moments induced by vector leptoquarks at one loop. We find that $O(1) \mathrm{CP}$-violating phases in tau and muon couplings can lead to corresponding EDMs within reach of next-generation EDM experiments, and that existing bounds on the electron EDM already put stringent constraints on CP-violating electron couplings.

Keywords: Beyond Standard Model, CP violation, Heavy Quark Physics

ARXIV EPRINT: 2002.01400 


\section{Contents}

1 Introduction 1

2 The CP violating $U_{1}$ vector leptoquark model 3

2.1 Leptoquark effects in B-meson decays 4

3 Dipole moments of quarks and leptons $\quad 6$

3.1 Leptoquark contribution to dipole moments of SM leptons and quarks 6

3.2 Connecting quark dipole moments to the neutron EDM 8

3.3 Experimental status and prospects 9

4 Flavor anomalies and electric dipole moments $\quad 10$

$\begin{array}{ll}\text { 4.1 Probing the parameter space using tau measurements } & 10\end{array}$

$\begin{array}{lll}4.2 & \text { Probing the parameter space using muon measurements } & 13\end{array}$

$\begin{array}{lll}4.3 & \text { Probing the parameter space using electron measurements } & 15\end{array}$

$\begin{array}{lll}5 & \text { LHC bounds on the leptoquark } & 17\end{array}$

$\begin{array}{lll}6 & \text { Conclusions } & 19\end{array}$

\section{Introduction}

Over the past several years, multiple $B$-physics experiments, including BaBar, LHCb, and Belle, have reported anomalies in decays associated with the $b \rightarrow c \ell \nu$ and $b \rightarrow s \ell \ell$ transitions. Violations of lepton flavor universality (LFU), known to be theoretically clean probes of New Physics (NP), are of particular interest. In the Standard Model (SM) LFU is only broken by the lepton masses. Hints for additional sources of LFU violation have been observed in the ratios of branching ratios of flavor-changing charged current and neutral current decays of $B$ mesons, $R_{D}, R_{D^{*}}, R_{K}$, and $R_{K^{*}}$,

$$
R_{D^{(*)}}=\frac{\mathrm{BR}\left(B \rightarrow D^{(*)} \tau \nu\right)}{\mathrm{BR}\left(B \rightarrow D^{(*)} \ell \nu\right)}, \quad R_{K^{(*)}}=\frac{\mathrm{BR}\left(B \rightarrow K^{(*)} \mu^{+} \mu^{-}\right)}{\mathrm{BR}\left(B \rightarrow K^{(*)} e^{+} e^{-}\right)} .
$$

The experimental world averages of $R_{D}$ and $R_{D^{*}}$ from the heavy flavor averaging group (HFLAV) are based on measurements from BaBar [1], Belle [2-4], and LHCb [5, 6], and read $[7]$

$$
R_{D}=0.340 \pm 0.027 \pm 0.013, \quad R_{D^{*}}=0.295 \pm 0.011 \pm 0.008,
$$

with an error correlation of $\rho=-38 \%$. The corresponding SM predictions are known with high precision [8-10]. The values adopted by HFLAV are [7]

$$
R_{D}^{\mathrm{SM}}=0.299 \pm 0.003, \quad R_{D^{*}}^{\mathrm{SM}}=0.258 \pm 0.005 .
$$


The combined discrepancy between the SM prediction and experimental world averages of $R_{D}$ and $R_{D^{*}}$ is at the $3.1 \sigma$ level.

The most precise measurement to date of the LFU ratio $R_{K}$ has been performed by $\mathrm{LHCb}[11]$

$$
R_{K}=0.846_{-0.054-0.014}^{+0.060+0.016}, \quad \text { for } 1.1 \mathrm{GeV}^{2}<q^{2}<6 \mathrm{GeV}^{2}
$$

with $q^{2}$ being the dilepton invariant mass squared. The SM predicts $R_{K}^{\mathrm{SM}} \simeq 1$ with theoretical uncertainties well below the current experimental ones [12]. The above experimental value is closer to the SM prediction than the Run-1 result [13]. However, the reduced experimental uncertainties still imply a tension between theory and experiment of $2.5 \sigma$.

The most precise measurement of $R_{K^{*}}$ is from a Run-1 LHCb analysis [14] that finds

$$
R_{K^{*}}= \begin{cases}0.66_{-0.07}^{+0.11} \pm 0.03, & \text { for } 0.045 \mathrm{GeV}^{2}<q^{2}<1.1 \mathrm{GeV}^{2} \\ 0.69_{-0.07}^{+0.11} \pm 0.05, & \text { for } 1.1 \mathrm{GeV}^{2}<q^{2}<6 \mathrm{GeV}^{2}\end{cases}
$$

The result for both $q^{2}$ bins are in tension with the SM prediction [12], $R_{K^{*}}^{\mathrm{SM}} \simeq 1$, by $\sim 2.5 \sigma$ each. Recent measurements of $R_{K^{*}}$ and $R_{K}$ by Belle $[15,16]^{1}$

$$
\begin{gathered}
R_{K^{*}}= \begin{cases}0.90_{-0.21}^{+0.27} \pm 0.10, & \text { for } 0.1 \mathrm{GeV}^{2}<q^{2}<8 \mathrm{GeV}^{2}, \\
1.18_{-0.32}^{+0.52} \pm 0.10, & \text { for } 15 \mathrm{GeV}^{2}<q^{2}<19 \mathrm{GeV}^{2},\end{cases} \\
R_{K}= \begin{cases}0.98_{-0.23}^{+0.27} \pm 0.06, & \text { for } 1 \mathrm{GeV}^{2}<q^{2}<6 \mathrm{GeV}^{2}, \\
1.11_{-0.26}^{+0.29} \pm 0.07, & \text { for } 14.18 \mathrm{GeV}^{2}<q^{2},\end{cases}
\end{gathered}
$$

are compatible with both the SM prediction and the LHCb results. Several papers have re-analyzed the status of the $B$ anomalies in light of the latest experimental updates, and found preference for new physics with high significance [17-23].

While the anomalies detailed upon above persist, the question of the origin of the observed baryon asymmetry [24] also remains a long standing problem in cosmology. Any dynamical explanation requires sizable $\mathrm{C}$ - and $\mathrm{CP}$-violating interactions in the early universe [25]. In light of upcoming low-energy experiments with much greater sensitivity to electric and magnetic dipole moments of elementary particles, it is interesting to ask whether solutions to the flavor anomalies may also be associated with sizable CP violating complex phases that may be probed by these experiments.

The only known viable, single-mediator explanation of all flavor anomalies is a $U_{1}$ vector leptoquark [26-32]. This leptoquark generically introduces new sources of CP violation in the Lagrangian in the form of complex parameters [33]. The scope of the present study is to explore, for the first time, the prospects of observing electric dipole moments (EDMs) induced by a $U_{1}$ vector leptoquark that could explain the flavor anomalies reviewed above. We additionally explore collider constraints, as well as constraints from measurements of the magnetic moments, and other flavor observables. Implications for EDMs and other CPV observables in scalar leptoquark scenarios have recently been discussed in [34-40].

\footnotetext{
${ }^{1}$ Here we quote the isospin average of $B^{0} \rightarrow K^{(*) 0} \ell^{+} \ell^{-}$and $B^{ \pm} \rightarrow K^{(*) \pm} \ell^{+} \ell^{-}$decays.
} 
This paper is organized as follows: in section 2, we introduce the $\mathrm{CP}$ violating $U_{1}$ model and discuss its effects on the B-physics anomalies. In section 3, we give an overview of the effects of the CP violating leptoquark on EDMs of quarks, leptons, and neutrons. We also include a discussion of the present status of the experimental searches and the prospects for future measurements. In section 4, we report the main results of our paper, showing the leptoquark parameter space that can be probed by B-physics and EDM measurements. In section 5, we discuss the LHC bounds on our leptoquark model. Finally, we reserve section 6 for our conclusions.

\section{The CP violating $U_{1}$ vector leptoquark model}

We consider the vector leptoquark $U_{1}=(\mathbf{3}, \mathbf{1})_{2 / 3}$ (triplet under $\mathrm{SU}(3)_{c}$, singlet under $\mathrm{SU}(2)_{L}$, and with hypercharge $\left.+2 / 3\right)$. This model may be viewed as the low energy limit of Pati-Salam models described in refs. [41, 42] (see also [43-50]). The most general dimension-4 Lagrangian describing the vector leptoquark of mass $M_{U_{1}}$ is (see e.g. [51] for a recent review)

$$
\begin{aligned}
\mathcal{L}_{U_{1}}= & -\frac{1}{2} U_{\mu \nu}^{\dagger} U^{\mu \nu}+M_{U_{1}}^{2} U_{\mu}^{\dagger} U^{\mu} \\
& +i g_{s} U_{\mu}^{\dagger} T_{a} U_{\nu}\left(\kappa_{s} G_{a}^{\mu \nu}+\tilde{\kappa}_{s} \tilde{G}_{a}^{\mu \nu}\right)+i g^{\prime} \frac{2}{3} U_{\mu}^{\dagger} U_{\nu}\left(\kappa_{Y} B^{\mu \nu}+\tilde{\kappa}_{Y} \tilde{B}^{\mu \nu}\right), \\
& +\sum_{i, j}\left(\lambda_{i j}^{q}\left(\bar{Q}_{i} \gamma_{\mu} P_{L} L_{j}\right) U^{\mu}+\lambda_{i j}^{d}\left(\bar{D}_{i} \gamma_{\mu} P_{R} E_{j}\right) U^{\mu}\right)+\text { h.c. },
\end{aligned}
$$

where $U^{\mu \nu}=D^{\mu} U^{\nu}-D^{\nu} U^{\mu}$ is the leptoquark field strength tensor in terms of its vector potential $U^{\mu}$ and gauge covariant derivative $D^{\mu}=\partial^{\mu}+i g_{s} T_{a} G_{a}^{\mu}+i g^{\prime} \frac{2}{3} B^{\mu}$. $G_{a}^{\mu}$ and $B^{\mu}$, and $G_{a}^{\mu \nu}$ and $B^{\mu \nu}$ are the gluon and hypercharge vector potentials and field strengths, respectively. The dual field strength tensors are $\tilde{G}_{a}^{\mu \nu}=\frac{1}{2} \epsilon^{\mu \nu \rho \sigma} G_{a \rho \sigma}$ and $\tilde{B}^{\mu \nu}=\frac{1}{2} \epsilon^{\mu \nu \rho \sigma} B_{\rho \sigma}$.

The third line in eq. (2.1) contains couplings of $U_{1}$ with the SM quarks and leptons. Specifically, $Q_{i}$ and $L_{i}$ are the left-handed quark and lepton doublets, while $D_{j}$ and $E_{j}$ are the right-handed down quark and charged lepton singlets. We assume that the model does not contain light right-handed neutrinos. (If right-handed neutrinos are introduced, additional couplings of $U_{1}$ with right-handed neutrinos and right-handed up quarks are possible [52].) The couplings $\lambda_{i j}^{q}$ and $\lambda_{i j}^{d}$ are in general complex and are therefore a potential source of $\mathrm{CP}$ violation of the model. We work in the fermion mass eigenstate basis and define the leptoquark couplings $\lambda_{i j}^{q}$ and $\lambda_{i j}^{d}$ in a way such that

$$
\mathcal{L}_{U_{1}} \supset \sum_{i j k}\left(V_{i k} \lambda_{k j}^{q}\right)\left(\bar{u}_{i} \gamma_{\mu} P_{L} \nu_{j}\right) U^{\mu}+\sum_{i j} \lambda_{i j}^{q}\left(\bar{d}_{i} \gamma_{\mu} P_{L} \ell_{j}\right) U^{\mu}+\sum_{i j} \lambda_{i j}^{d}\left(\bar{d}_{i} \gamma_{\mu} P_{R} \ell_{j}\right) U^{\mu}+\text { h.c. },
$$

where $V$ is the CKM matrix.

The second line in eq. (2.1) encodes the chromo- and hypercharge- magnetic and electric dipole moments of the $U_{1}$ leptoquark.

If the leptoquark arises from the spontaneous breakdown of a gauge symmetry, gauge invariance requires these couplings to be fixed to $\kappa_{s}=\kappa_{Y}=1, \tilde{\kappa}_{s}=\tilde{\kappa}_{Y}=0$. In more 
generic scenarios where $U_{1}$ is composite, the values of $\kappa_{s}, \tilde{\kappa}_{s}, \kappa_{Y}, \tilde{\kappa}_{Y}$ are free parameters. Non-zero values for $\tilde{\kappa}_{s}$ and $\tilde{\kappa}_{Y}$ are an additional potential source of CP violation. However, since they do not directly influence flavor physics, we will focus our attention to CPviolation contained in $\lambda_{i j}^{q}$ and $\lambda_{i j}^{d}$ (even though in section 3 we will present fully generic expressions for the EDMs, including their dependence on $\tilde{\kappa}_{s}$ and $\tilde{\kappa}_{Y}$ ).

\subsection{Leptoquark effects in B-meson decays}

The $U_{1}$ leptoquark can simultaneously address the hints for LFU violation in charged current decays $R_{D^{(*)}}$ and in neutral current decays $R_{K^{(*)}} .{ }^{2}$ Here we will use the results of a recent study [21] that identified a benchmark point in the leptoquark parameter space that gives a remarkably consistent new physics explanation of these hints. We will explore the parameter space around this benchmark point (supplemented by a few more points), focusing on the implications for dipole moments. As we discuss below, not all leptoquark couplings in (2.1) are required to address the anomalies.

Explaining the observed values of $R_{D^{(*)}}$ by non-standard effects in the $b \rightarrow c \tau \nu$ transition is possible if the leptoquark has sizable couplings to the left-handed tau. Avoiding strong constraints from leptonic tau decays $\tau \rightarrow \nu_{\tau} \ell \bar{\nu}_{\ell}$ and the $B \rightarrow X_{s} \gamma$ decay is possible in a well defined parameter space around the benchmark point with $\lambda_{33}^{q} \simeq 0.7, \lambda_{23}^{q} \simeq 0.6$ with a leptoquark mass of $M_{U_{1}}=2 \mathrm{TeV}$ [21]. This corresponds to the following non-standard value for $R_{D^{(*)}}$

$$
\frac{R_{D^{(*)}}}{R_{D^{(*)}}^{\mathrm{SM}}}=\left|1+\frac{v^{2}}{2 M_{U_{1}}^{2}} \frac{\lambda_{33}^{q *} \lambda_{23}^{q}}{V_{c s} V_{c b}}\right|^{2} \simeq 1.2,
$$

which is in good agreement with observations (in this equation we normalize $v=246 \mathrm{GeV}$ ).

The results for $R_{K^{(*)}}$ can be accommodated by a non-standard effect in the $b \rightarrow s \mu \mu$ transition if the couplings to the left-handed muon obey $\operatorname{Re}\left(\lambda_{22}^{q} \times \lambda_{32}^{q}\right) \simeq-2.5 \times 10^{-3}$ for $M_{U_{1}}=2 \mathrm{TeV}$ [21]. The leptoquark effects for this choice of couplings are described by a shift in the Wilson coefficients of the effective Hamiltonian relevant for $b \rightarrow$ sll transitions (see e.g. [21] for the precise definition)

$$
C_{9}^{b s \mu \mu}=-C_{10}^{b s \mu \mu}=-\frac{4 \pi^{2}}{e^{2}} \frac{v^{2}}{M_{U_{1}}^{2}} \frac{\lambda_{32}^{q} \lambda_{22}^{q *}}{V_{t s}^{*} V_{t b}} \simeq-0.4
$$

This agrees well with the best fit value for the Wilson coefficients found in [21].

The muonic couplings $\lambda_{22}^{q}, \lambda_{32}^{q}$ (that can explain the $R_{K^{(*)}}$ anomalies) in combination with the tauonic couplings $\lambda_{23}^{q}, \lambda_{33}^{q}$ (that are required to explain the $R_{D^{(*)}}$ anomalies) lead to lepton flavor violating decays. The strongest constraints arise from the decays $\tau \rightarrow \phi \mu$ and $B \rightarrow K \tau \mu$. For the $\lambda_{33}^{q}, \lambda_{23}^{q}$ benchmark mentioned above, existing limits on those decay modes result in the bounds on the leptoquark couplings $\left|\lambda_{22}^{q}\right| \lesssim 0.16$ and $\left|\lambda_{32}^{q}\right| \lesssim 0.40$ for $M_{U_{1}}=2 \mathrm{TeV}[21]$.

The experimental values of $R_{K^{(*)}}$ may also be explained by new physics in the $b \rightarrow$ see transition as opposed to modifying the $b \rightarrow s \mu \mu$ transition. Focusing on left-handed

\footnotetext{
${ }^{2}$ Note that the small anomaly in the low $q^{2}$ bin of $R_{K}^{*}$ in (1.5) cannot be fully addressed by the $U_{1}$ leptoquark, but it requires the presence of light NP [53-56].
} 
couplings, the required shifts in the relevant Wilson coefficients is [21]

$$
C_{9}^{b s e e}=-C_{10}^{b s e e}=-\frac{4 \pi^{2}}{e^{2}} \frac{v^{2}}{M_{U_{1}}^{2}} \frac{\lambda_{31}^{q} \lambda_{21}^{q *}}{V_{t s}^{*} V_{t b}} \simeq+0.4,
$$

corresponding to the couplings $\operatorname{Re}\left(\lambda_{21}^{q} \times \lambda_{31}^{q}\right) \simeq+2.5 \times 10^{-3}$ for $M_{U_{1}}=2 \mathrm{TeV}$. The experimental bounds on the lepton flavor violating processes $\tau \rightarrow \phi e$ and $B \rightarrow K \tau e$ are comparable to those of $\tau \rightarrow \phi \mu$ and $B \rightarrow K \tau \mu$ [57-59]. We therefore expect that the constraints on the left-handed electron couplings $\left|\lambda_{21}^{q}\right|$ and $\left|\lambda_{31}^{q}\right|$ are similar to the muon couplings mentioned above, i.e. $\left|\lambda_{21}^{q}\right| \lesssim 0.16$ and $\left|\lambda_{31}^{q}\right| \lesssim 0.40$ for $M_{U_{1}}=2 \mathrm{TeV}$.

Motivated by this discussion, in the next sections we will explore the leptoquark parameter space in the neighborhood of four benchmark scenarios:

$$
\begin{array}{ll}
\text { BM1: } & \lambda_{33}^{q}=0.7, \quad \lambda_{23}^{q}=0.6, \quad \lambda_{32}^{q}=-0.25, \quad \lambda_{22}^{q}=0.01, \quad \lambda_{31}^{q}=\lambda_{21}^{q}=0, \\
\text { BM2: } & \lambda_{33}^{q}=0.7, \quad \lambda_{23}^{q}=0.6, \quad \lambda_{32}^{q}=\lambda_{22}^{q}=0, \quad \lambda_{31}^{q}=0.05, \lambda_{21}^{q}=0.05, \\
\text { BM3: } & \lambda_{33}^{q}=\lambda_{23}^{q}=0, \quad \lambda_{32}^{q}=-1.4, \quad \lambda_{22}^{q}=10^{-3}, \quad \lambda_{31}^{q}=\lambda_{21}^{q}=0, \\
\text { BM4: } & \lambda_{33}^{q}=\lambda_{23}^{q}=0, \quad \lambda_{32}^{q}=\lambda_{22}^{q}=0, \quad \lambda_{31}^{q}=0.5, \quad \lambda_{21}^{q}=5.0 \times 10^{-3}, \\
& M_{U_{1}}=2 \mathrm{TeV}, \quad \kappa_{Y, s}=1, \quad \tilde{\kappa}_{Y, s}=0 \text { for all benchmarks }
\end{array}
$$

with all the other fermionic couplings of the leptoquark in eq. (2.1) set to zero. In BM1 and BM2 both the $R_{D^{(*)}}$ and $R_{K^{(*)}}$ anomalies are addressed. The $R_{K^{(*)}}$ explanations involve new physics in the $b \rightarrow s \mu \mu$ transition (BM1) or in the $b \rightarrow$ see transition (BM2). For benchmark points BM3 and BM4 we forgo an explanation of $R_{D^{(*)}}$. This allows us to increase the couplings to muons/electrons while avoiding the strong constraints from lepton flavor violating tau decays. Note that in benchmark BM3, the $R_{K^{(*)}}$ anomalies are only partially addressed. For BM3 we have $R_{K} \simeq R_{K}^{*} \simeq 0.88$ which is in good agreement with the latest $R_{K}$ measurement, but $\sim 2 \sigma$ away from the measured $R_{K^{*}}$ value. As we discuss below in section 4.2 benchmark BM3 is motivated because it can accommodate the longstanding discrepancy in the anomalous magnetic moment of the muon.

For all benchmark scenarios we explicitly checked compatibility with the measurements of the di-lepton [60] and di-tau [61] invariant mass distributions at the LHC and searches for electron-quark contact interactions at LEP [62]. In the case of the di-lepton invariant mass distributions at the LHC, the value of $\lambda_{32}^{q}$ in BM3 is close to the exclusion bound.

Starting with these benchmark points, in the following sections we turn on couplings to right-handed taus $\lambda_{33}^{d}$, muons $\lambda_{32}^{d}$, and electrons $\lambda_{31}^{d}$ and determine the expected size of electric and magnetic dipole moments of the leptons as function of the real and imaginary part of the new couplings. In principle, the couplings $\lambda_{23}^{d}, \lambda_{22}^{d}$ and $\lambda_{21}^{d}$ will also influence the dipole moments; we comment on $\lambda_{22}^{d}$ and $\lambda_{21}^{d}$ in sections 4.2 and 4.3, but we do not consider $\lambda_{23}^{d}$ since it does not play any role in explaining the flavor anomalies. The couplings $\lambda_{3 i}^{d}$ mentioned above do modify the new physics contributions to the flavor anomalies. However, as we will discuss below in section 4, once the existing constraints on those couplings from other flavor observables are taken into account, the effect on the flavor anomalies turns out to be small. 

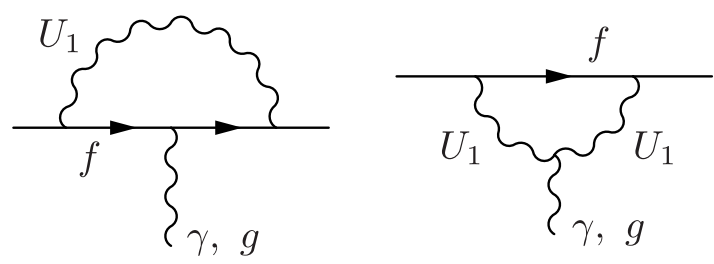

Figure 1. Feynman diagrams contributing to the dipole moments of quarks and leptons from leptoquark exchange.

\section{Dipole moments of quarks and leptons}

In this section, we calculate and present new and original formulae for shifts in the electric and magnetic dipole moments of leptons and quarks induced by the leptoquark. We then estimate the size of the neutron electric dipole. Finally, we review experimental limits on the dipole moments.

The leptoquark radiatively induces dipole moments starting at one loop order as shown in figure 1. After integrating out the leptoquark, effective interactions encoding the dipole moments are given by the effective Lagrangian

$$
\mathcal{L}_{\text {eff }}=\sum_{f}\left(a_{f} \frac{e Q_{f}}{4 m_{f}}\left(\bar{f} \sigma^{\mu \nu} f\right) F_{\mu \nu}-\frac{i d_{f}}{2}\left(\bar{f} \sigma^{\mu \nu} \gamma_{5} f\right) F_{\mu \nu}\right),
$$

where $a_{f}$ is the anomalous magnetic dipole moment, and $d_{f}$ is the electric dipole moment of SM fermion $f$. In the absence of right-handed neutrinos, the $U_{1}$ leptoquark does not generate dipole moments for neutrinos.

Through its coupling with the gluons, the leptoquark induces chromomagnetic, $\hat{a}_{q}$, and chromoelectric, $\hat{d}_{q}$, dipole moments of quarks

$$
\mathcal{L}_{\mathrm{eff}}=\sum_{q}\left(\frac{\hat{a}_{q}}{4 m_{q}}\left(\bar{q} \sigma^{\mu \nu} T^{a} q\right) G_{\mu \nu}^{a}-\frac{i \hat{d}_{q}}{2}\left(\bar{q} \sigma^{\mu \nu} T^{a} \gamma_{5} q\right) G_{\mu \nu}^{a}\right) .
$$

\subsection{Leptoquark contribution to dipole moments of SM leptons and quarks}

In the large $M_{U_{1}}$ limit, the leptoquark contribution to the anomalous magnetic moment of the muon is

$$
\begin{aligned}
a_{\mu}= & \frac{N_{C}}{16 \pi^{2}} \sum_{i}\left[2 \operatorname{Re}\left(\lambda_{i 2}^{q} \lambda_{i 2}^{d *}\right) \frac{m_{d_{i}} m_{\mu}}{M_{U_{1}}^{2}}\left(2 Q_{d}+Q_{U}\left(\left(1-\kappa_{Y}\right) \ln \left(\frac{\Lambda_{\mathrm{UV}}^{2}}{M_{U_{1}}^{2}}\right)+\frac{1-5 \kappa_{Y}}{2}\right)\right)\right. \\
& +2 Q_{U} \tilde{\kappa}_{Y} \operatorname{Im}\left(\lambda_{i 2}^{q} \lambda_{i 2}^{d *}\right) \frac{m_{d_{i}} m_{\mu}}{M_{U_{1}}^{2}}\left(\ln \left(\frac{\Lambda_{\mathrm{UV}}^{2}}{M_{U_{1}}^{2}}\right)+\frac{5}{2}\right) \\
& \left.-\left(\left|\lambda_{i 2}^{q}\right|^{2}+\left|\lambda_{i 2}^{d}\right|^{2}\right) \frac{m_{\mu}^{2}}{M_{U_{1}}^{2}}\left(\frac{4}{3} Q_{d}+Q_{U}\left(\left(1-\kappa_{Y}\right) \ln \left(\frac{\Lambda_{\mathrm{UV}}^{2}}{M_{U_{1}}^{2}}\right)-\frac{1+9 \kappa_{Y}}{6}\right)\right)\right],
\end{aligned}
$$

where $Q_{d}=-1 / 3, Q_{U}=+2 / 3$ is the leptoquark electric charge, and $N_{C}=3$. Our formula is in agreement with $[63,64]$ when specialized to the vector leptoquark model with $\kappa_{Y}=1$ and $\tilde{\kappa}_{Y}=0$. Note that if $\kappa_{Y} \neq 1$ or $\tilde{\kappa}_{Y} \neq 0$, relevant for scenarios in which the leptoquark is 
not a gauge boson, the dipole moment exhibits logarithmic dependence on the cut-off scale $\Lambda_{U V}$ not far above the leptoquark mass. This cut-off dependence signals the presence of additional contributions in UV complete scenarios (e.g. from other resonances in a strongly coupled model.) Even in the case that the leptoquark is a gauge boson, and the expressions that we derive are thus formally UV finite, we would like to remark that UV models will likely contain additional contributions to EDMs e.g. from an extended Higgs sector.

Similarly, the muon electric dipole moment is

$$
\begin{aligned}
d_{\mu}= & \frac{e N_{C}}{16 \pi^{2}} \sum_{i}\left[\operatorname{Im}\left(\lambda_{i 2}^{q} \lambda_{i 2}^{d *}\right) \frac{m_{d_{i}}}{M_{U_{1}}^{2}}\left(2 Q_{d}+Q_{U}\left(\left(1-\kappa_{Y}\right) \ln \left(\frac{\Lambda_{\mathrm{UV}}^{2}}{M_{U_{1}}^{2}}\right)+\frac{1-5 \kappa_{Y}}{2}\right)\right)\right. \\
& +Q_{U} \tilde{\kappa}_{Y} \operatorname{Re}\left(\lambda_{i 2}^{q} \lambda_{i 2}^{d *}\right) \frac{m_{d_{i}}}{M_{U_{1}}^{2}}\left(\ln \left(\frac{\Lambda_{\mathrm{UV}}^{2}}{M_{U_{1}}^{2}}\right)+\frac{5}{2}\right) \\
& \left.+Q_{U} \tilde{\kappa}_{Y}\left(\left|\lambda_{i 2}^{q}\right|^{2}+\left|\lambda_{i 2}^{d}\right|^{2}\right) \frac{m_{\mu}}{M_{U_{1}}^{2}}\left(\frac{1}{2} \ln \left(\frac{\Lambda_{\mathrm{UV}}^{2}}{M_{U_{1}}^{2}}\right)+\frac{3}{4}\right)\right] .
\end{aligned}
$$

$\mathrm{CP}$ violation is provided either by the imaginary part of the fermion coupling combination $\lambda_{i 2}^{q} \lambda_{i 2}^{d *}$, or by the CP violating hypercharge coupling $\tilde{\kappa}_{Y}$. Dipole moments of other charged leptons are obtained by the appropriate replacement of the muon mass, $m_{\mu}$, and leptoquark couplings to muons, $\lambda_{i 2}$.

The bottom quark electric dipole moment induced by the leptoquark is

$$
\begin{aligned}
d_{b}= & \frac{e}{16 \pi^{2}} \sum_{i}\left[\operatorname{Im}\left(\lambda_{3 i}^{q} \lambda_{3 i}^{d *}\right) \frac{m_{\ell_{i}}}{M_{U_{1}}^{2}}\left(2 Q_{\ell}+Q_{U}\left(\left(1-\kappa_{Y}\right) \ln \left(\frac{\Lambda_{\mathrm{UV}}^{2}}{M_{U_{1}}^{2}}\right)+\frac{1-5 \kappa_{Y}}{2}\right)\right)\right. \\
& +Q_{U} \tilde{\kappa}_{Y} \operatorname{Re}\left(\lambda_{3 i}^{q} \lambda_{3 i}^{d *}\right) \frac{m_{\ell_{i}}}{M_{U_{1}}^{2}}\left(\ln \left(\frac{\Lambda_{\mathrm{UV}}^{2}}{M_{U_{1}}^{2}}\right)+\frac{5}{2}\right) \\
& \left.-Q_{U} \tilde{\kappa}_{Y}\left(\left|\lambda_{3 i}^{q}\right|^{2}+\left|\lambda_{3 i}^{d}\right|^{2}\right) \frac{m_{b}}{M_{U_{1}}^{2}}\left(\frac{1}{2} \ln \left(\frac{\Lambda_{\mathrm{UV}}^{2}}{M_{U_{1}}^{2}}\right)+\frac{3}{4}\right)\right]
\end{aligned}
$$

and the chromoelectric dipole moment (cEDM) is

$$
\begin{aligned}
\hat{d}_{b}= & \frac{g_{s}}{16 \pi^{2}} \sum_{i}\left[\operatorname{Im}\left(\lambda_{3 i}^{q} \lambda_{3 i}^{d *}\right) \frac{m_{\ell_{i}}}{M_{U_{1}}^{2}}\left(\left(1-\kappa_{Y}\right) \ln \left(\frac{\Lambda_{\mathrm{UV}}^{2}}{M_{U_{1}}^{2}}\right)+\frac{1-5 \kappa_{Y}}{2}\right)\right. \\
& +\tilde{\kappa}_{Y} \operatorname{Re}\left(\lambda_{3 i}^{q} \lambda_{3 i}^{d *}\right) \frac{m_{\ell_{i}}}{M_{U_{1}}^{2}}\left(\ln \left(\frac{\Lambda_{\mathrm{UV}}^{2}}{M_{U_{1}}^{2}}\right)+\frac{5}{2}\right) \\
& \left.-\tilde{\kappa}_{Y}\left(\left|\lambda_{3 i}^{q}\right|^{2}+\left|\lambda_{3 i}^{d}\right|^{2}\right) \frac{m_{b}}{M_{U_{1}}^{2}}\left(\frac{1}{2} \ln \left(\frac{\Lambda_{\mathrm{UV}}^{2}}{M_{U_{1}}^{2}}\right)+\frac{3}{4}\right)\right] .
\end{aligned}
$$

The other down-type quark (chromo-)electric dipole moments can be obtained by appropriate replacements of flavor indices.

Analogously, up-type quark (chromo-)electric dipole moments are obtained from the bottom quark result by the replacement $\lambda_{i j}^{q} \rightarrow V_{i k} \lambda_{k j}^{q}, \lambda_{i j}^{d} \rightarrow 0, m_{\ell} \rightarrow m_{\nu}=0, m_{b} \rightarrow m_{u}$ yielding

$$
d_{u}=-\frac{e}{16 \pi^{2}} Q_{U} \tilde{\kappa}_{Y} \sum_{i}\left|\left(V \lambda^{q}\right)_{1 i}\right|^{2} \frac{m_{u}}{M_{U_{1}}^{2}}\left(\frac{1}{2} \ln \left(\frac{\Lambda_{\mathrm{UV}}^{2}}{M_{U_{1}}^{2}}\right)+\frac{3}{4}\right) \text {, }
$$


and

$$
\hat{d}_{u}=-\frac{g_{s}}{16 \pi^{2}} \tilde{\kappa}_{Y} \sum_{i}\left|\left(V \lambda^{q}\right)_{1 i}\right|^{2} \frac{m_{u}}{M_{U_{1}}^{2}}\left(\frac{1}{2} \ln \left(\frac{\Lambda_{\mathrm{UV}}^{2}}{M_{U_{1}}^{2}}\right)+\frac{3}{4}\right) .
$$

We do not consider anomalous (chromo-)magnetic moments of the quarks as they are hardly constrained by experiment. Note that the anomalous magnetic moments of the top quark is constrained by measurements of $t \bar{t}$ production at the LHC. Current bounds of $\hat{a}_{t} \sim 0.1[65]$ are, however, not sensitive to the effects induced by heavy leptoquark loops in our scenario.

\subsection{Connecting quark dipole moments to the neutron EDM}

In the following, we determine the neutron electric dipole moment due to quark-level dipole moments. We neglect the running of quark dipole moments from the leptoquark scale to the hadronic scale, since the neglected logarithm of order $\alpha_{s} \ln \left(M_{U_{1}}^{2} / M_{n}^{2}\right) \approx 1.6$ leads to corrections which are small compared to the relevant hadronic uncertainties discussed below.

The dominant contributions to the neutron EDM are from the short range QCD interactions involving quark EDMs, $d_{i}$, and cEDMs, $\hat{d}_{i}$, given by

$$
d_{n} \sim-\frac{v}{\sqrt{2}}\left[\beta_{n}^{u G} \hat{d}_{u}+\beta_{n}^{d G} \hat{d}_{d}+\beta_{n}^{s G} \hat{d}_{s}+\beta_{n}^{u \gamma} d_{u}+\beta_{n}^{d \gamma} d_{d}+\beta_{n}^{s \gamma} d_{s}\right],
$$

where the $\beta_{i}^{(k)}$ are the hadronic matrix elements. Estimates from quark cEDM are given by $\beta_{n}^{u G} \approx 4_{-3}^{+6} \times 10^{-4} e \mathrm{fm}$ and $\beta_{n}^{d G} \approx 8_{-6}^{+10} \times 10^{-4} e \mathrm{fm}$ [66]. The most recent lattice evaluations of the matrix elements involving the electromagnetic EDMs are [67, 68] $-\frac{v}{\sqrt{2}} \beta_{n}^{u \gamma} \approx-0.233(28),-\frac{v}{\sqrt{2}} \beta_{n}^{d \gamma} \approx 0.776(66)$ and $-\frac{v}{\sqrt{2}} \beta_{n}^{s \gamma} \approx 0.008(9)$.

Contributions from heavy quark $\mathrm{cEDM}$ are estimated by integrating out the heavy quark, $Q=c, b$, to generate the three gluon Weinberg (gluon cEDM) operator,

$$
\mathcal{L}=\frac{c_{\tilde{\mathrm{G}}}}{m_{Q}^{2}} \frac{g_{s} f^{a b c}}{3} \tilde{G}_{\mu \nu}^{a} G_{\nu \rho}^{b} G_{\rho}^{c \mu},
$$

where the Wilson coefficient is given by [69-71]

$$
c_{\tilde{\mathrm{G}}}=\frac{g_{s}^{2}}{32 \pi^{2}} m_{Q} \hat{d}_{Q} .
$$

Contributions to $c_{\tilde{\mathrm{G}}}$ from CP-violating leptoquark gluon interactions proportional to $\tilde{\kappa}_{s}$ are also present, but we do not consider them since they are unrelated to flavor anomalies. In terms of $c_{\tilde{\mathrm{G}}}$, the neutron EDM is given by [66]

$$
d_{n}=\frac{v^{2}}{m_{Q}^{2}} \beta_{n}^{\tilde{G}} c_{\tilde{\mathrm{G}}}
$$

where $\beta_{n}^{\tilde{G}} \approx[2,40] \times 10^{-20}$ e cm is the nucleon matrix element estimated using QCD sum rules and chiral perturbation theory $[72,73]$. 
To compare the relative sizes of contributions from light and heavy quark to the neutron EDM, we take the strange and bottom quark contributions, and assume for simplicity that $\kappa_{Y}=1, \tilde{\kappa}_{Y}=0$. We also assume $M_{U_{1}} \sim 2 \mathrm{TeV}$ for the leptoquark scale.

Putting together eqs. (3.5) and (3.6) with eq. (3.9), we find that the strange quark EDM contribution to the neutron EDM is

$$
\begin{aligned}
d_{n}^{\text {strange }} & \approx-\frac{5}{24 \pi^{2} M_{U_{1}}^{2} \mathrm{~cm}}\left[m_{\tau} \operatorname{Im}\left(\lambda_{23}^{q} \lambda_{23}^{d *}\right)+m_{\mu} \operatorname{Im}\left(\lambda_{22}^{q} \lambda_{22}^{d *}\right)\right] \times 0.008 e \mathrm{~cm} \\
& \sim-\left(\operatorname{Im}\left(\lambda_{23}^{q} \lambda_{23}^{d *}\right)+0.06 \operatorname{Im}\left(\lambda_{22}^{q} \lambda_{22}^{d *}\right)\right) \times 1.5 \times 10^{-24} e \mathrm{~cm} .
\end{aligned}
$$

The bottom quark cEDM contribution to the neutron EDM is instead given by

$$
\begin{aligned}
d_{n}^{\text {bottom }} & \approx-\frac{g_{s}^{3} v^{2}}{\left(16 \pi^{2}\right)^{2} m_{b} M_{U_{1}}^{2}}\left[m_{\tau} \operatorname{Im}\left(\lambda_{33}^{q} \lambda_{33}^{d *}\right)+m_{\mu} \operatorname{Im}\left(\lambda_{32}^{q} \lambda_{32}^{d *}\right)\right] \times[2,40] \times 10^{-20} \\
& \sim-\left(\operatorname{Im}\left(\lambda_{33}^{q} \lambda_{33}^{d *}\right)+0.06 \operatorname{Im}\left(\lambda_{32}^{q} \lambda_{32}^{d *}\right)\right) \times[2,40] \times 5 \times 10^{-27} \text { e cm }
\end{aligned}
$$

For generic $\mathcal{O}(1)$ sized leptoquark couplings $\lambda_{i k}^{q}$ and $\lambda_{i k}^{d}$ the strange quark contribution (3.13) to the neutron EDM is much larger than the bottom quark contribution (3.14). However, in the region of parameter space we are exploring, the bottom quark contribution is typically bigger than the strange quark contribution.

\subsection{Experimental status and prospects}

We review here the current experimental status of dipole moments of Standard Model fermions. The anomalous magnetic moments of the electron, $a_{e}$, and the muon, $a_{\mu}$, are measured extremely precisely [74, 75], and are predicted to similarly high precision within the SM, with new physics contributions constrained to lie within the range [76, 77] (see also [78-80])

$$
\Delta a_{\mu}=\left(28.0 \pm 6.3_{\exp } \pm 3.8_{\mathrm{th}}\right) \times 10^{-10}, \quad \Delta a_{e}=\left(-8.9 \pm 3.6_{\exp } \pm 2.3_{\mathrm{th}}\right) \times 10^{-13},
$$

In addition to the long standing discrepancy in the muon magnetic moment with a significance of more than $3 \sigma$, a discrepancy in the electron magnetic moment arose after a recent precision measurement of the fine structure constant [81] with a significance of $\sim 2.4 \sigma$. Combining the expected sensitivity from the running $g-2$ experiment at Fermilab [82] with expected progress on the SM prediction (see [83-88] for recent lattice efforts and [8993] for recent efforts using the framework of dispersion relations) the uncertainty on $\Delta a_{\mu}$ will be reduced by a factor of a few in the coming years. Similarly, for $\Delta a_{e}$ we expect an order of magnitude improvement in the sensitivity [94].

The anomalous magnetic moment of the tau, $a_{\tau}$, is currently only very weakly constrained. The strongest constraint comes from LEP and reads at 95\% C.L. [95]

$$
-0.055<a_{\tau}<0.013
$$

Improvements in sensitivity by an order of magnitude or more might be achieved at Belle II or future electron positron colliders (see [96] for a review). 
Strong experimental constraints exist for the EDM of the electron. The strongest bound is inferred from the bound on the EDM of ThO obtained by the ACME collaboration which gives at $90 \%$ C.L. [97]

$$
\left|d_{e}\right|<1.1 \times 10^{-29} e \mathrm{~cm} .
$$

Significant improvements by an order of magnitude or more can be expected from ACME in the future [97].

Only weak constraints exist for the EDMs of the muon and the tau, $d_{\mu}$ and $d_{\tau}$. Analyses by the Muon g-2 collaboration [98] and the Belle collaboration [99] give the following bounds at $95 \%$ C.L.

$$
\left|d_{\mu}\right|<1.9 \times 10^{-19} e \mathrm{~cm}, \quad-2.2 \times 10^{-17} e \mathrm{~cm}<d_{\tau}<4.5 \times 10^{-17} e \mathrm{~cm} .
$$

The proposed muon EDM experiment at PSI aims at improving the sensitivity to the muon EDM by 4 orders of magnitude, $d_{\mu} \lesssim 5 \times 10^{-23} e \mathrm{~cm}$ [100]. Improving the sensitivity to the tau EDM by roughly two orders of magnitude $\left(d_{\tau}<2 \times 10^{-19} \mathrm{e} \mathrm{cm}\right)$ might be possible at Belle II or at future $e^{+} e^{-}$colliders [101].

Turning to quarks, we note that the magnetic and chromo-magnetic dipole moments of quarks, $a_{q}$ and $\hat{a}_{q}$, are very weakly constrained and we therefore do not consider them in this work. As discussed in the previous section, the EDMs and cEDMs of quarks, $d_{q}$ and $\hat{d}_{q}$, lead to EDMs of hadronic systems like the neutron and are therefore strongly constrained. In the following we will focus on the neutron EDM which is bounded at 90\% C.L. by [102]

$$
\left|d_{n}\right|<1.8 \times 10^{-26} e \mathrm{~cm} .
$$

Experimental sensitivities should improve by two orders of magnitude to a few $10^{-28} e \mathrm{~cm}$ in the next decade [103].

We collect the SM predictions, the current experimental results, and expected future experimental sensitivities to the dipole moments in table 1 .

\section{Flavor anomalies and electric dipole moments}

In this section, we study the impact of leptoquarks on (c)EDMs and $B$-physics measurements at the benchmark points presented in section 2.1.

\subsection{Probing the parameter space using tau measurements}

Given the BM1 and BM2 benchmarks for the leptoquark couplings to left-handed taus, $\lambda_{33}^{q} \simeq 0.7, \lambda_{23}^{q} \simeq 0.6$, we begin by turning on the coupling to right-handed taus $\lambda_{33}^{d}$ while setting the right-handed couplings to muons and electrons ( $\lambda_{32}^{d}$ and $\lambda_{31}^{d}$, respectively) to zero. The coupling $\lambda_{33}^{d}$ will induce the dipole moments of the tau as in eqs. (3.3) and (3.4), as well as transition dipole moments leading to the lepton flavor violating decay modes $\tau \rightarrow \mu \gamma$ and $\tau \rightarrow e \gamma$. In the limit $m_{e}, m_{\mu} \ll m_{\tau} \ll m_{b}$, the partial width for the $U_{1}$ contribution to $\tau \rightarrow \mu \gamma$ is given by

$$
\begin{aligned}
\Gamma_{\tau \rightarrow \mu \gamma}= & \frac{\alpha m_{\tau}^{3} N_{C}^{2}}{256 \pi^{4} M_{U_{1}}^{4}} m_{b}^{2}\left|\lambda_{32}^{q} \lambda_{33}^{d *}\right|^{2}\left[\left(2 Q_{b}-Q_{U}\left(\left(1-\kappa_{Y}\right) \ln \left(\frac{\Lambda_{\mathrm{UV}}^{2}}{M_{U_{1}}^{2}}\right)+\frac{1-5 \kappa_{Y}}{2}\right)\right)^{2}\right. \\
& \left.+Q_{U}^{2} \tilde{\kappa}_{Y}^{2}\left(\ln \left(\frac{\Lambda_{\mathrm{UV}}^{2}}{M_{U_{1}}^{2}}\right)+\frac{5}{2}\right)^{2}\right]
\end{aligned}
$$




\begin{tabular}{|cccc|}
\hline observable & SM theory & current exp. & projected sens. \\
\hline$a_{e}-a_{e}^{\mathrm{SM}}$ & $\pm 2.3 \times 10^{-13}[76,81]$ & $(-8.9 \pm 3.6) \times 10^{-13}[74]$ & $\sim 10^{-14}[94]$ \\
$a_{\mu}-a_{\mu}^{\mathrm{SM}}$ & $\pm 3.8 \times 10^{-10}[76]$ & $(28.0 \pm 6.3) \times 10^{-10}[75]$ & $1.6 \times 10^{-10}[82]$ \\
$a_{\tau}-a_{\tau}^{\mathrm{SM}}$ & $\pm 3.9 \times 10^{-8}[76]$ & $(-2.1 \pm 1.7) \times 10^{-2}[95]$ & \\
\hline$d_{e}$ & $<10^{-44} e \mathrm{~cm}[104,105]$ & $<1.1 \times 10^{-29} e \mathrm{~cm}[97]$ & $\sim 10^{-30} e \mathrm{~cm} \mathrm{[97]}$ \\
$d_{\mu}$ & $<10^{-42} e \mathrm{~cm}[105]$ & $<1.9 \times 10^{-19} e \mathrm{~cm} \mathrm{[98]}$ & $\sim 10^{-23} e \mathrm{~cm} \mathrm{[100]}$ \\
$d_{\tau}$ & $<10^{-41} e \mathrm{~cm}[105]$ & $(1.15 \pm 1.70) \times 10^{-17} e \mathrm{~cm}[99]$ & $\sim 10^{-19} e \mathrm{~cm} \mathrm{[101]}$ \\
\hline$d_{n}$ & $\sim 10^{-32} e \mathrm{~cm}[106]$ & $<1.8 \times 10^{-26} e \mathrm{~cm}[102]$ & few $\times 10^{-28} e \mathrm{~cm} \mathrm{[103]}$ \\
\hline
\end{tabular}

Table 1. Summary of Standard Model theory errors/bounds (first column), current experimental measurements/limits (second column) and projected precision of next-generation experiments (third column) of magnetic moment anomalies and electric dipole moments of the charged leptons and the neutron. For clarity, for the anomalous magnetic moments, the Standard Model central values have been subtracted. We are not aware of any experimental analysis for the projected sensitivity of the tau magnetic moment.

This expression is in agreement with [32], when specialized to the vector leptoquark model with $\kappa_{Y}=1$ and $\tilde{\kappa}_{Y}=0$. The expression for the decay mode $\tau \rightarrow e \gamma$ is obtained by an appropriate replacement of the lepton flavor index. The experimental upper limits on the branching ratios of the $\tau \rightarrow \mu \gamma$ and $\tau \rightarrow e \gamma$ decays are $5.0 \times 10^{-8}$ and $5.4 \times 10^{-8}$, respectively [7].

In addition to inducing lepton flavor violating tau decays, the $\lambda_{33}^{d}$ coupling will modify the new physics contributions to charged current decays based on the $b \rightarrow c \tau \nu$ and $b \rightarrow u \tau \nu$ transitions and neutral current decays based on $b \rightarrow s \tau \tau$. The decay modes that are particularly sensitive to right-handed currents are the helicity suppressed two body decays $B_{c} \rightarrow \tau \nu[107,108], B^{ \pm} \rightarrow \tau \nu$, and $B_{s} \rightarrow \tau^{+} \tau^{-}$. We find

$$
\begin{aligned}
& \frac{\mathrm{BR}\left(B_{c} \rightarrow \tau \nu\right)}{\operatorname{BR}\left(B_{c} \rightarrow \tau \nu\right)_{\mathrm{SM}}}=\left|1-\frac{\sum_{j} V_{c j} \lambda_{j 3}^{q}}{V_{c b}} \frac{v^{2}}{M_{U_{1}}^{2}}\left(\frac{\lambda_{33}^{q *}}{2}+\frac{\lambda_{33}^{d *} m_{B_{c}}^{2}}{m_{\tau}\left(m_{b}+m_{c}\right)}\right)\right|^{2}, \\
& \frac{\operatorname{BR}\left(B^{ \pm} \rightarrow \tau \nu\right)}{\operatorname{BR}\left(B^{ \pm} \rightarrow \tau \nu\right)_{\mathrm{SM}}}=\left|1-\frac{\sum_{j} V_{u j} \lambda_{j 3}^{q}}{V_{u b}} \frac{v^{2}}{M_{U_{1}}^{2}}\left(\frac{\lambda_{33}^{q *}}{2}+\frac{\lambda_{33}^{d *} m_{B^{ \pm}}^{2}}{m_{\tau} m_{b}}\right)\right|^{2},
\end{aligned}
$$

Using the expression for the branching ratio in terms of the Wilson coefficients from [109], we find

$$
\begin{aligned}
\frac{\mathrm{BR}\left(B_{s} \rightarrow \tau^{+} \tau^{-}\right)}{\operatorname{BR}\left(B_{s} \rightarrow \tau^{+} \tau^{-}\right)_{\mathrm{SM}}}= & \left|1+\frac{4 \pi^{2}}{e^{2} C_{10}^{\mathrm{SM}}} \frac{v^{2}}{M_{U_{1}}^{2}}\left(\frac{\lambda_{33}^{q *} \lambda_{23}^{q}+\lambda_{33}^{d *} \lambda_{23}^{d}}{V_{t s}^{*} V_{t b}}-\frac{m_{B_{s}}^{2}}{m_{\tau} m_{b}} \frac{\lambda_{33}^{q *} \lambda_{23}^{d}+\lambda_{33}^{d *} \lambda_{23}^{q}}{V_{t s}^{*} V_{t b}}\right)\right|^{2} \\
& +\frac{16 \pi^{4}}{e^{4}\left(C_{10}^{\mathrm{SM}}\right)^{2}} \frac{v^{4}}{M_{U_{1}}^{4}} \frac{m_{B_{s}}^{4}}{m_{\tau}^{2} m_{b}^{2}}\left|\frac{\lambda_{33}^{q *} \lambda_{23}^{d}-\lambda_{33}^{d *} \lambda_{23}^{q}}{V_{t s}^{*} V_{t b}}\right|^{2}\left(1-\frac{4 m_{\tau}^{2}}{m_{B_{s}}^{2}}\right)
\end{aligned}
$$

where we neglected the finite life time difference in the $B_{s}$ system. We use a normalization such that the SM value for the Wilson coefficient is $C_{10}^{\mathrm{SM}} \simeq-4.1$ [110]. Renormalization group running from the leptoquark scale down to the $b$-scale can be incorporated by evaluating the quark masses in eqs. (4.2)-(4.4) at the scale $\mu \simeq 2 \mathrm{TeV}$. Note that the terms 
containing both left-handed and right-handed couplings enjoy a mild chiral enhancement by factors $m_{B_{c}}^{2} /\left(m_{\tau}\left(m_{b}+m_{c}\right)\right), m_{B^{ \pm}}^{2} /\left(m_{\tau} m_{b}\right)$, and $m_{B_{s}}^{2} /\left(m_{\tau} m_{b}\right)$, respectively.

The measured $\operatorname{BR}\left(B^{ \pm} \rightarrow \tau \nu\right)=(1.09 \pm 0.24) \times 10^{-4}$ [111] agrees well with the SM prediction $\mathrm{BR}\left(B^{ \pm} \rightarrow \tau \nu\right)_{\mathrm{SM}}=\left(0.838_{-0.029}^{+0.039}\right) \times 10^{-4}[112]$, yielding

$$
\frac{\mathrm{BR}\left(B^{ \pm} \rightarrow \tau \nu\right)}{\mathrm{BR}\left(B^{ \pm} \rightarrow \tau \nu\right)_{\mathrm{SM}}}=1.30 \pm 0.29
$$

So far no direct measurement of the $B_{c} \rightarrow \tau \nu$ branching ratio has been performed. We impose the bound $\mathrm{BR}\left(B_{c} \rightarrow \tau \nu\right)<30 \%$ [108]. The SM branching ratio is

$$
\mathrm{BR}\left(B_{c} \rightarrow \tau \nu\right)_{\mathrm{SM}}=\tau_{B_{c}} m_{B_{c}} \frac{f_{B_{c}}^{2} G_{F}^{2}}{8 \pi}\left|V_{c b}\right|^{2} m_{\tau}^{2}\left(1-\frac{m_{\tau}^{2}}{m_{B_{c}}^{2}}\right)^{2}=(2.21 \pm 0.09) \times 10^{-2},
$$

with the lifetime of the $B_{c}$ meson $\tau_{B_{c}}=(0.507 \pm 0.009) \times 10^{-12} \mathrm{~s}[111]$, the $B_{c}$ decay constant $f_{B_{c}}=(0.427 \pm 0.006) \mathrm{GeV}[113]$ and we used $\left|V_{c b}\right|=(41.6 \pm 0.56) \times 10^{-3}[112]$.

Similarly, the $B_{s} \rightarrow \tau^{+} \tau^{-}$decay has not been observed so far. The first direct limit on the branching ratio was placed by $\mathrm{LHCb}[114]$ and is $\operatorname{BR}\left(B_{s} \rightarrow \tau^{+} \tau^{-}\right)<6.8 \times 10^{-3}$, while the SM branching ratio is $\operatorname{BR}\left(B_{s} \rightarrow \tau^{+} \tau^{-}\right)_{\mathrm{SM}}=(7.73 \pm 0.49) \times 10^{-7}$ [115]. The projected sensitivity $\mathrm{BR}\left(B_{s} \rightarrow \tau^{+} \tau^{-}\right) \sim 5 \times 10^{-4}$ from LHCb with $50 \mathrm{fb}^{-1}$ [116].

In figure 2, we show current and projected constraints on the $U_{1}$ leptoquark in the plane of the complex $\lambda_{33}^{d}$ coupling divided by the leptoquark mass for BM1 and BM2 benchmark points. The figure represents both BM1 and BM2, since the shown constraints are independent of the muon couplings $\lambda_{32}^{q}, \lambda_{22}^{q}$ and electron couplings $\lambda_{31}^{q}, \lambda_{21}^{q}$ and changing from BM1 to BM2 does not affect our results. The most stringent constraint comes from $B_{s} \rightarrow \tau^{+} \tau^{-}$and is depicted by the gray shaded region in the figure. The projected sensitivity of LHCb to $B_{s} \rightarrow \tau^{+} \tau^{-}$is indicated by the dashed gray curve. Constraints from $B^{ \pm} \rightarrow \tau \nu, B_{c} \rightarrow \tau \nu$, and lepton flavor violating tau decays $(\tau \rightarrow \mu \gamma$ for benchmark BM1 and $\tau \rightarrow e \gamma$ for BM2) are slightly weaker and exclude values of $\lambda_{33}^{d}$ that are a factor of a few larger than those excluded by $B_{s} \rightarrow \tau^{+} \tau^{-}$. (In figure 2 we show only the strongest constraint coming from $B_{s} \rightarrow \tau^{+} \tau^{-}$.) Once the bounds are imposed, the allowed values of the right-handed coupling $\lambda_{33}^{d}$ are sufficiently small such that they do not affect $R_{D^{(*)}}, R_{K^{(*)}}$ in a significant way. Therefore, in all the allowed region in figure 2 , the anomalies are satisfied.

From the figure, we observe that the current experimental bounds on $d_{\tau}$ and $a_{\tau}$ do not constraint the parameter space in a relevant way. The constraint from $a_{\tau}$ is depicted by the red hatched region in figure 2 , while the experimental bound on $d_{\tau}$ constrains values of $\Im\left(\lambda_{33}^{d}\right) / M_{U_{1}}$ that are $\mathcal{O}\left(10^{5}\right) \mathrm{TeV}^{-1}$, and, therefore, beyond the range of the plot. Projected sensitivities of next-generation experiments to the tau EDM [101] (shown by the dashed blue line) are still far from being able to probe the viable new physics parameter space.

In addition to the tau electric and anomalous magnetic dipole moments, the $U_{1}$ leptoquark coupling, $\lambda_{33}^{d}$, will contribute to the neutron EDM, $d_{n}$. The constraint from the current bound on the neutron EDM is shown by the solid purple line in figure 2, where the region above this line is excluded due to the leptoquark generating a contribution to 


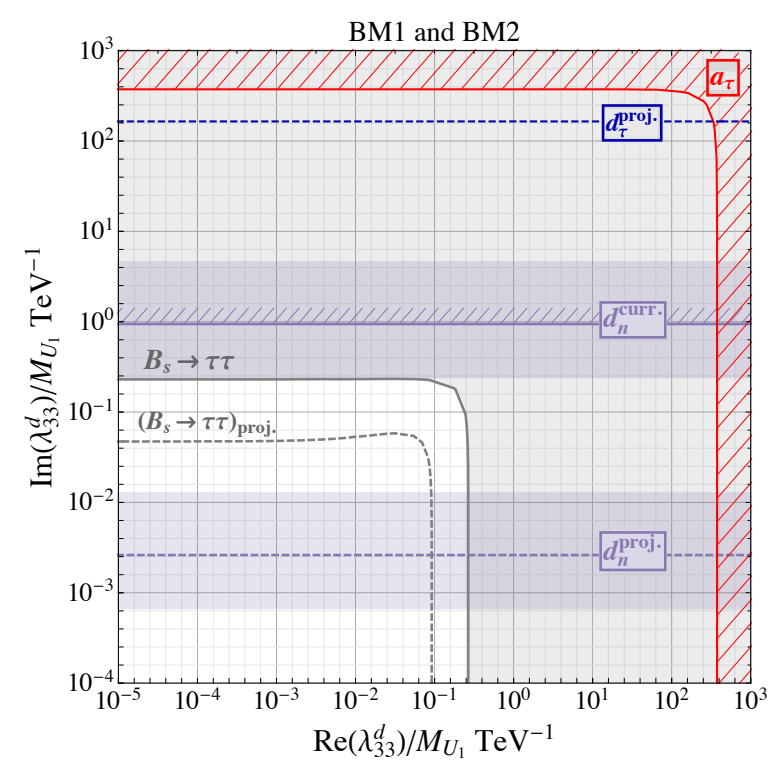

Figure 2. Constraints on the $U_{1}$ leptoquark parameter space in the plane of the complex coupling $\lambda_{33}^{d}$ divided by the leptoquark mass, $M_{U_{1}}$, and all other parameters fixed as in BM1 (2.6a) or BM2 (2.6b). The gray region enclosed by the solid gray curve represents parameter space that is excluded by $B_{s} \rightarrow \tau^{+} \tau^{-}$, while the dashed gray curve is the projected sensitivity of LHCb to $B_{s} \rightarrow \tau^{+} \tau^{-}$. The red hatched region is excluded by the bound on the tau lepton anomalous magnetic moment. The dashed blue line is the projected sensitivity of future experiments to the tau EDM. The region above the solid purple line is excluded by bounds on the neutron EDM, and the dashed purple line is the projected sensitivity of future neutron EDM experiments. The surrounding purple bands reflect the theoretical uncertainty in the nucleon matrix element $\beta_{n}^{\tilde{G}}$. Note that the observables shown in the figure are independent of $\lambda_{32}^{q}, \lambda_{22}^{q}$ and $\lambda_{31}^{q}, \lambda_{21}^{q}$, and the change from benchmark BM1 to BM2 has no effect on the exclusion curves.

the neutron EDM that is too large. The surrounding purple bands reflect the theoretical uncertainty in the nucleon matrix element $\beta_{n}^{\tilde{G}}$. We observe that the currend bound on the neutron EDM leads to a constraint that is weaker than $B_{s} \rightarrow \tau^{+} \tau^{-}$and is not yet probing the allowed parameter space. On the other hand, the projected sensitivity of future neutron EDM experiments [103] (shown by the dashed purple line) will begin probing the new physics parameter space and can lead to stronger constraints on the amount of $\mathrm{CP}$ violation present in the right-handed couplings of $U_{1}$ to tau leptons.

\subsection{Probing the parameter space using muon measurements}

Next we focus on the BM1 and BM3 benchmarks, and investigate the impact of the leptoquark couplings to right-handed muons, $\lambda_{32}^{d}$, while setting the right-handed tau and electron couplings ( $\lambda_{33}^{d}$ and $\lambda_{31}^{d}$, respectively) to zero. The coupling $\lambda_{32}^{d}$ will lead to a shift in the anomalous magnetic moment of the muon, $\Delta a_{\mu}$, in the muon EDM, $d_{\mu}$, and in the EDM of the bottom quark given in eqs. (3.3), (3.4), and (3.5), as well as the lepton flavor violating decay mode $\tau \rightarrow \mu \gamma$ given in eq. (4.1) with $\left|\lambda_{32}^{q} \lambda_{33}^{d *}\right|^{2} \rightarrow\left|\lambda_{32}^{d} \lambda_{33}^{q *}\right|^{2}$. In the presence of the coupling $\lambda_{32}^{d}$, the muon dipole moment enjoys a sizable chiral enhancement by $m_{b} / m_{\mu}$. 

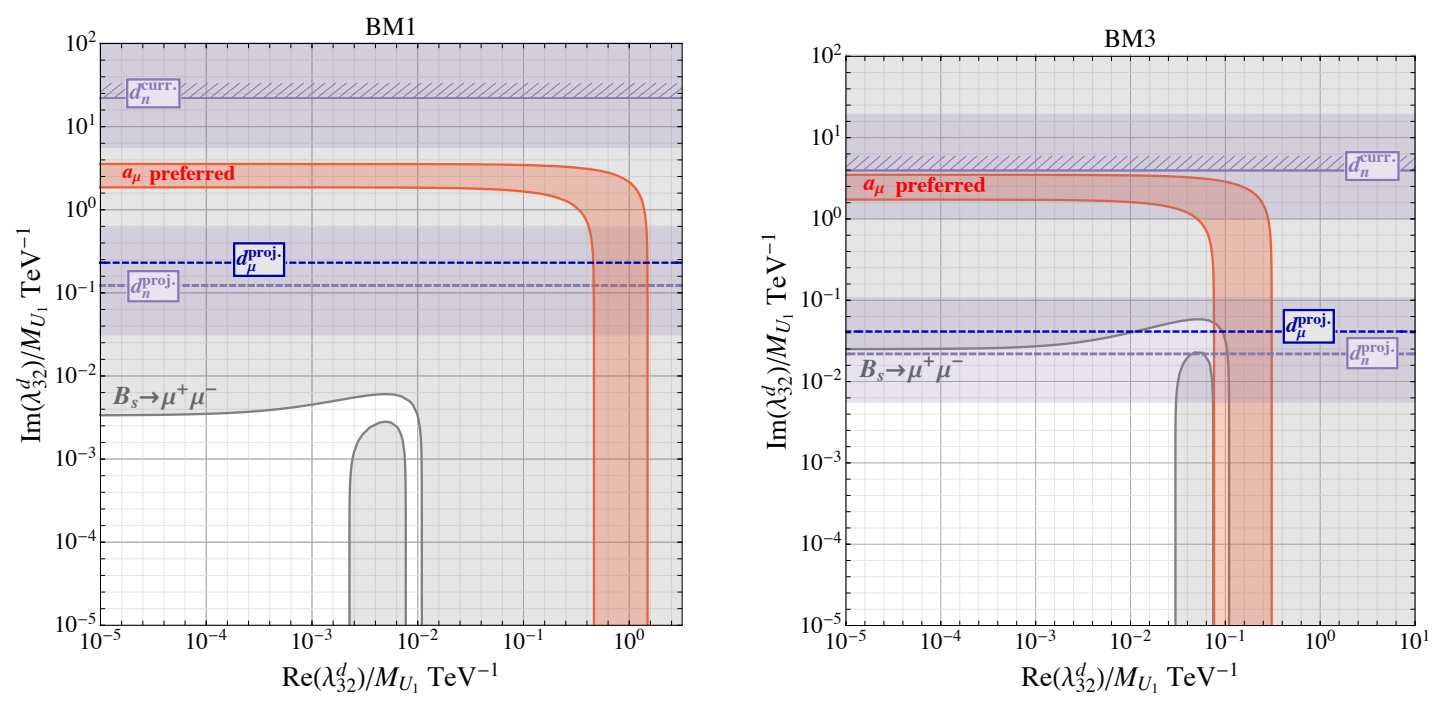

Figure 3. Constraints on the $U_{1}$ leptoquark parameter space in the plane of the complex $\lambda_{32}^{d}$ coupling divided by the leptoquark mass for the benchmark points BM1 (left panel) and BM3 (right panel). The gray region is excluded by $B_{s} \rightarrow \mu^{+} \mu^{-}$at the $95 \%$ C.L.. The dashed blue line is the projected sensitivity of future experiments to the muon EDM. The red shaded region corresponds to the parameter space the can address the anomaly in the anomalous magnetic moment of the muon. The solid (dashed) purple lines represent the current constraint (projected sensitivity) from the neutron EDM, with the purple bands reflecting the uncertainty in the nucleon matrix element $\beta_{n}^{\tilde{G}}$.

In addition, the coupling $\lambda_{32}^{d}$ can also give sizable non-standard effects in the $B_{s} \rightarrow$ $\mu^{+} \mu^{-}$decay. The corresponding expression is analogous to the one for the $B_{s} \rightarrow \tau^{+} \tau^{-}$ decay given in eq. (4.4)

$$
\begin{aligned}
\frac{\operatorname{BR}\left(B_{s} \rightarrow \mu^{+} \mu^{-}\right)}{\operatorname{BR}\left(B_{s} \rightarrow \mu^{+} \mu^{-}\right)_{\mathrm{SM}}}= & \left|1+\frac{4 \pi^{2}}{e^{2} C_{10}^{\mathrm{SM}}} \frac{v^{2}}{M_{U_{1}}^{2}}\left(\frac{\lambda_{32}^{q *} \lambda_{22}^{q}+\lambda_{32}^{d *} \lambda_{22}^{d}}{V_{t s}^{*} V_{t b}}-\frac{m_{B_{s}}^{2}}{m_{\mu} m_{b}} \frac{\lambda_{32}^{q *} \lambda_{22}^{d}+\lambda_{32}^{d *} \lambda_{22}^{q}}{V_{t s}^{*} V_{t b}}\right)\right|^{2} \\
& +\frac{16 \pi^{4}}{e^{4}\left(C_{10}^{\mathrm{SM}}\right)^{2}} \frac{v^{4}}{M_{U_{1}}^{4}} \frac{m_{B_{s}}^{4}}{m_{\mu}^{2} m_{b}^{2}}\left|\frac{\lambda_{32}^{q *} \lambda_{22}^{d}-\lambda_{32}^{d *} \lambda_{22}^{q}}{V_{t s}^{*} V_{t b}}\right|^{2}
\end{aligned}
$$

The terms that contain both left-handed and right-handed couplings are chirally enchanced by a factor $m_{B_{s}}^{2} /\left(m_{\mu} m_{b}\right)$.

The branching ratio $\mathrm{BR}\left(B_{s} \rightarrow \mu^{+} \mu^{-}\right)$has been measured at LHCb, CMS and ATLAS [117-120]. We use the average of these results from [21], that, combined with the SM prediction $[115,121]$, reads

$$
\frac{\mathrm{BR}\left(B_{s} \rightarrow \mu^{+} \mu^{-}\right)}{\operatorname{BR}\left(B_{s} \rightarrow \mu^{+} \mu^{-}\right)_{\mathrm{SM}}}=0.73_{-0.10}^{+0.13}
$$

which is in slight tension $(\sim 2 \sigma)$ with the SM prediction. Interestingly enough, in the region of parameter space where the couplings to left-handed muons $\lambda_{22}^{q}, \lambda_{32}^{q}$ provide an explanation of $R_{K^{(*)}}$, the tension in $B_{s} \rightarrow \mu^{+} \mu^{-}$is largely lifted. 
In figure 3 we show the current and projected constraints on the $U_{1}$ leptoquark for BM1 (left) and BM3 (right) in the plane of the complex coupling $\lambda_{32}^{d}$ divided by the leptoquark mass. For both benchmarks, the most stringent constraint arises from $B_{s} \rightarrow \mu^{+} \mu^{-}$. The region that is excluded at the $95 \%$ C.L. is shaded in gray. Once the constraints from $B_{s} \rightarrow \mu^{+} \mu^{-}$are imposed, the allowed values of $\lambda_{32}^{d}$ are sufficiently small that they do not affect $R_{K^{(*)}}$ in a significant way. The region that is shaded in red is the region of parameter space that is able to address the anomaly in the anomalous magnetic moment of the muon, while the blue dashed lines are the projected sensitivities of future experiments to the muon EDM. Similar to figure 2, the solid (dashed) purple line is the current constraint (projected sensitivity) of the neutron EDM. The current bound on the muon EDM, $d_{\mu}$, is very weak and constrains values of $\Im\left(\lambda_{32}^{d}\right) / M_{U_{1}}$ outside from the range of the plot $\left(\Im\left(\lambda_{32}^{d}\right) / M_{U_{1}} \sim \mathcal{O}\left(10^{3}\right) \mathrm{TeV}^{-1}\right.$ for BM1 and $\Im\left(\lambda_{32}^{d}\right) / M_{U_{1}} \sim \mathcal{O}\left(10^{2}\right) \mathrm{TeV}^{-1}$ for BM3).

In the left plot of figure 3 we observe that, once the constraints from $B_{s} \rightarrow \mu^{+} \mu^{-}$ is imposed, the BM1 benchmark cannot address the $a_{\mu}$ anomaly. We conclude that the $U_{1}$ leptoquark can not explain the $B$ anomalies and the $(g-2)_{\mu}$ anomaly simultaneously with the parameters fixed to those of BM1. This is mainly due to limits on lepton flavor violating decays $\tau \rightarrow \phi \mu$ and $B \rightarrow K \tau \mu$ that impose stringent constraints on the size of the left-handed muonic couplings $\lambda_{32}^{q}$ and $\lambda_{22}^{q}$ (see discussion in section 2.1).

In order to avoid these constraints, we can instead set the $U_{1}$ couplings to left-handed tau leptons, $\lambda_{33}^{q}$ and $\lambda_{23}^{q}$, to zero as in BM3 in (2.6c). The decay rates $\tau \rightarrow \phi \mu, B \rightarrow K \tau \mu$, and $\tau \rightarrow \mu \gamma$ mediated by $U_{1}$ then go to zero, allowing the muonic couplings $\lambda_{32}^{q}$ and $\lambda_{22}^{q}$ to have larger values. However, by switching off $\lambda_{33}^{q}$ and $\lambda_{23}^{q}$ we forgo an explanation of $R_{D^{(*)}}$.

In the right plot of figure 3 we show that, for BM3, the region of parameter space that can address the $a_{\mu}$ anomaly (the red shaded region) overlaps with the region of parameter space that is allowed by $B_{s} \rightarrow \mu^{+} \mu^{-}$, and the $U_{1}$ leptoquark can therefore address both the $(g-2)_{\mu}$ anomaly and (at least partially, cf. discussion in section 2.1) the $R_{K^{(*)}}$ anomalies. Finally, we notice that, for this benchmark, projected sensitivities to the neutron EDM might start to probe the viable parameter space.

We also explored the region of parameter space with nonzero $\lambda_{22}^{d}$ instead of $\lambda_{32}^{d}$. In this case, for BM1 and BM3, the neutron EDM is dominated by the strange quark contribution (3.13), so its projected sensitivity covers larger region of parameter space. However in this case, we did not find any viable region of parameter space explaining the anomaly in $a_{\mu}$.

\subsection{Probing the parameter space using electron measurements}

Instead of muon specific couplings that address the discrepancies in the LFU ratios $R_{K^{(*)}}$ by new physics that suppresses the $b \rightarrow s \mu \mu$ transitions, one can also entertain the possibility that new physics addresses the anomaly by enhancing the $b \rightarrow$ see transitions. This can be achieved with the leptoquark couplings $\lambda_{31}^{d}, \lambda_{21}^{d}$ as given in eq. (2.5) and by our benchmark points BM2 and BM4.

These couplings will also lead to shifts in the anomalous magnetic moment of the electron, $\Delta a_{e}$, and, in the presence of CP violation, induce an electron EDM, $d_{e}$, (see eqs. (3.3) and (3.4), respectively), and the lepton flavor violating mode $\tau \rightarrow e \gamma$ (see eq. (4.1) 

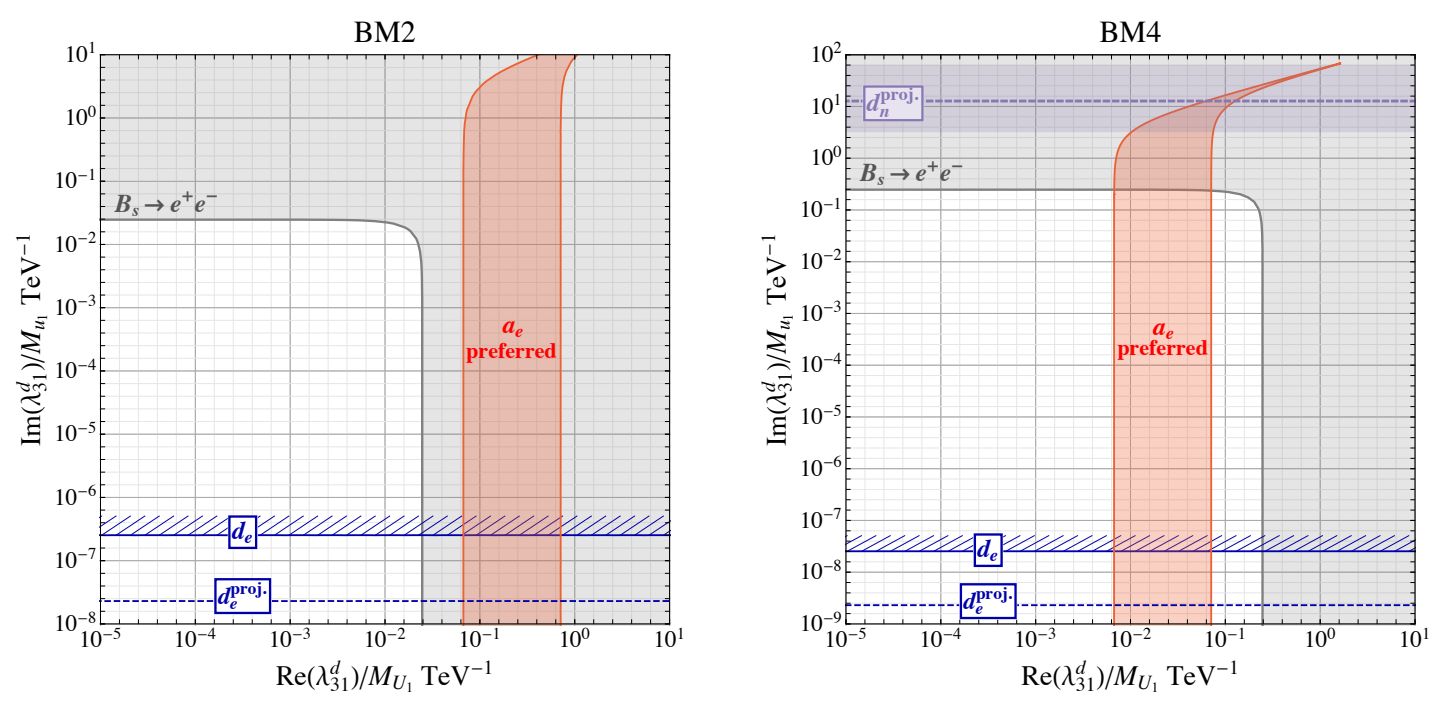

Figure 4. Constraints on the $U_{1}$ leptoquark parameter space in the plane of the complex coupling $\lambda_{31}^{d}$ divided by the leptoquark mass for the benchmark points BM2 and BM4, left and right panel, respectively). The gray region is excluded by $B_{s} \rightarrow e^{+} e^{-}$at the $95 \%$ C.L.. The red shaded region corresponds to the parameter space the can address the anomaly in the anomalous magnetic moment of the electron. The solid (dashed) blue lines represent the current constraint (projected sensitivity) from the electron EDM. In the right panel, the dashed purple line represents the projected sensitivity from the neutron EDM, with the purple band reflecting the uncertainty in the nucleon matrix element $\beta_{n}^{\tilde{G}}$.

with $\left.\left|\lambda_{32}^{q} \lambda_{33}^{d *}\right|^{2} \rightarrow\left|\lambda_{31}^{d} \lambda_{33}^{q *}\right|^{2}\right)$. Note that the chiral enhancement of the dipole moments $m_{b} / m_{e}$ can be particularly pronounced in the case of the electron.

In this scenario, potentially important constraints arise from the $B_{s} \rightarrow e^{+} e^{-}$decay. The effect of the leptoquark is given by an expression analogous to eq. (4.7) with $m_{\mu} \rightarrow m_{e}$ and $\lambda_{32}^{f}, \lambda_{22}^{f} \rightarrow \lambda_{31}^{f}, \lambda_{21}^{f}$, with the $\mathrm{SM}$ prediction given by $\mathrm{BR}\left(B_{s} \rightarrow e^{+} e^{-}\right)=(8.54 \pm 0.55) \times$ $10^{-14}$ [115]. Experimentally, the $B_{s} \rightarrow e^{+} e^{-}$branching ratio is bounded at the 90\% C.L. by $[122]$

$$
\operatorname{BR}\left(B_{s} \rightarrow e^{+} e^{-}\right)<2.8 \times 10^{-7} .
$$

The plots in figure 4 show the current and projected constraints on the $U_{1}$ leptoquark in the plane of the complex coupling $\lambda_{31}^{d}$ divided by the leptoquark mass for BM2 (left) and BM4 (right). In both panels the gray region is excluded by the bound from $B_{s} \rightarrow e^{+} e^{-}$, while the red shaded region is the region of parameter space that can address the $2.4 \sigma$ anomaly in the electron magnetic moment, $a_{e}$. The blue solid (dashed) lines are the current constraint (projected sensitivity) of the electron electric dipole moment, $d_{e}$. In the right panel, the dashed purple line and the surrounding purple band is the projected sensitivity of the neutron EDM, $d_{n}$.

For BM2 (left plot of figure 4) we observe that the region of parameter space that is able to address the anomaly in $a_{e}$ is excluded by constraints from $B_{s} \rightarrow e^{+} e^{-}$and a simultaneous explanation of all the $B$ anomalies and $a_{e}$ is not possible. This is due to 
stringent constraints on the size of $\lambda_{31}^{q}$ from the lepton flavor violating decays $\tau \rightarrow \phi e$ and $B \rightarrow K \tau e$ (see discussion in section 2.1). Constrains from the $\tau \rightarrow e \gamma$ are slightly weaker.

To avoid the stringent constraints from lepton flavor violating decays, we can set all the $U_{1}$ couplings to tau leptons to zero. Then, the $\tau \rightarrow \phi e$ and $B \rightarrow K \tau e$ rates as well as the $\tau \rightarrow e \gamma$ rate go to zero, and the left-handed couplings to electrons can be larger. However, by setting $\lambda_{33}^{q}$ and $\lambda_{23}^{q}$ to zero, we forgo an explanation of $R_{D^{(*)}}$. This scenario is given by BM4, and the resulting constraints are shown in the right plot of figure 4 . We observe that the smaller value of $\lambda_{21}^{q}=0.005$ in BM4 leads to weaker constraints on $\lambda_{31}^{d}$ from $B_{s} \rightarrow e^{+} e^{-}$. In addition, the larger value of $\lambda_{31}^{q}=0.5$ generates a larger contribution to the electron magnetic moment necessary to explain the slight tension in $a_{e}$. In moving from BM2 to BM4 the bound from $B_{s} \rightarrow e^{+} e^{-}$opens up a wide region in parameter space favorable for the electron magnetic moment, $a_{e}$. We conclude that BM4 can address the anomalies in both $R_{K^{(*)}}$ and $a_{e}$.

We also investigated the region of parameter space with nonzero $\lambda_{21}^{d}$ instead of $\lambda_{31}^{d}$. We find in BM2 and BM4 that sensitivity to $d_{e}$ is reduced because it is chirally enhanced by $m_{s}$ rather than $m_{b}$ in eq. (3.4). We also find no region of parameter space where the $U_{1}$ leptoquark explains the tension of the measured $a_{e}$ with theory.

\section{LHC bounds on the leptoquark}

Low-energy flavor observables like those discussed in the previous sections provide an indirect probe of the $U_{1}$ leptoquark. A complementary approach to probe the existence of $U_{1}$ is direct production at high energy colliders and looking for signatures of their decay products. The goal of this section is to compute the lower bound on the leptoquark mass in the allowed regions of parameter space in figures $2-4$.

The two main production mechanisms are single production in association with a lepton $\left(g q \rightarrow \ell U_{1}\right)$, and pair production $\left(g g, q \bar{q} \rightarrow U_{1} \overline{U_{1}}\right)$. For a recent review see [129, 130]. Once produced, the leptoquark will decay into a pair of SM fermions. The interactions of the $U_{1}$ leptoquark with SM quarks and leptons in eq. (2.2) generate the decays of $U_{1}$ into an up-type quark and a neutrino, or a down-type quark and a charged lepton. In the limit where $M_{U_{1}}$ is much larger than the masses of the decay products, the partial widths of $U_{1}$ are given by

$$
\begin{aligned}
& \Gamma\left(U_{1} \rightarrow u_{i} \nu_{j}\right)=\frac{M_{U_{1}}}{24 \pi}\left|\sum_{k=1,2,3} V_{i k} \lambda_{k j}^{q}\right|^{2}, \\
& \Gamma\left(U_{1} \rightarrow d_{i} \ell_{j}\right)=\frac{M_{U_{1}}}{24 \pi}\left(\left|\lambda_{i j}^{q}\right|^{2}+\left|\lambda_{i j}^{d}\right|^{2}\right),
\end{aligned}
$$

where $i, j=1,2,3$ label the three generations.

Several dedicated searches for singly and pair produced scalar leptoquarks have been performed by the LHC, and are classified according to whether the leptoquark decays to first, second, or third generation fermions. The strongest bounds on leptoquark pairproduction from ATLAS and CMS have been compiled in table 2, where the searches are organized according to whether the branching ratio into a quark and a charged lepton 


\begin{tabular}{|c|c|c|}
\hline \multicolumn{3}{|c|}{ LHC Bounds on Scalar Leptoquarks } \\
\hline Channel & Experiment & Limit \\
\hline \multicolumn{3}{|c|}{ First Generation Leptoquarks } \\
\hline $\operatorname{eejj}(\beta=1)$ & $\begin{array}{c}\text { ATLAS [123] } \\
\text { CMS [124] }\end{array}$ & $\begin{array}{l}1400 \mathrm{GeV} \\
1435 \mathrm{GeV}\end{array}$ \\
\hline$e \nu j j(\beta=0.5)$ & $\begin{array}{c}\text { ATLAS [123] } \\
\text { CMS [124] }\end{array}$ & $\begin{array}{l}1290 \mathrm{GeV} \\
1270 \mathrm{GeV}\end{array}$ \\
\hline \multicolumn{3}{|c|}{ Second Generation Leptoquarks } \\
\hline$\mu \mu j j(\beta=1)$ & $\begin{array}{c}\text { ATLAS [123] } \\
\text { CMS [125] }\end{array}$ & $\begin{array}{l}1560 \mathrm{GeV} \\
1530 \mathrm{GeV}\end{array}$ \\
\hline$\mu \nu j j(\beta=0.5)$ & $\begin{array}{l}\text { ATLAS [123] } \\
\text { CMS [125] }\end{array}$ & $\begin{array}{l}1230 \mathrm{GeV} \\
1285 \mathrm{GeV}\end{array}$ \\
\hline \multicolumn{3}{|c|}{ Third Generation Leptoquarks } \\
\hline \multirow{2}{*}{$b \tau b \tau$} & ATLAS [126] & $1030 \mathrm{GeV}$ \\
\hline & CMS [127] & $1020 \mathrm{GeV}$ \\
\hline \multicolumn{3}{|c|}{ Reinterpreted SUSY searches } \\
\hline$q \nu q \nu$ & CMS [128] & $980 \mathrm{GeV}$ \\
\hline$t \nu t \nu$ & $\begin{array}{l}\text { ATLAS [126] } \\
\text { CMS [128] }\end{array}$ & $\begin{array}{l}1000 \mathrm{GeV} \\
1020 \mathrm{GeV}\end{array}$ \\
\hline \multicolumn{3}{|c|}{ LHC Bounds on Vector Leptoquarks } \\
\hline Channel & Experiment & Limit \\
\hline \multicolumn{3}{|c|}{ Reinterpreted SUSY searches } \\
\hline$q \nu q \nu$ & CMS [128] & $\begin{array}{l}1410 \mathrm{GeV}\left(\kappa_{s}=0\right) \\
1790 \mathrm{GeV}\left(\kappa_{s}=1\right)\end{array}$ \\
\hline$t \nu t \nu$ & CMS [128] & $\begin{array}{l}1460 \mathrm{GeV}\left(\kappa_{s}=0\right) \\
1780 \mathrm{GeV}\left(\kappa_{s}=1\right)\end{array}$ \\
\hline
\end{tabular}

Table 2. LHC bounds on pair-production of scalar and vector leptoquarks. For scalar leptoquarks, the first three sections correspond to bounds from dedicated leptoquark searches, while the last section corresponds to bounds derived from the reinterpretation of squark pair production searches. For vector leptoquarks, only reintepreted SUSY searches exist. The parameter $\beta$ denotes the branching ratio of the leptoquark to a quark and a charged lepton. We do not report the bounds on the decays of the LQ to down-type quarks and a neutrino since these decays do not exist in our model.

(denoted by $\beta$ ) is $100 \%$ or $50 \%$, with the remaining $50 \%$ to a quark and a neutrino. In addition, in the table we also report the CMS reinterpretation of the squark pair production searches to place constraints on pair produced vector leptoquarks decaying to a quark and a neutrino, $t \nu$, or $q \nu(q=u, c, d, s)$ [128]. Similarly, ATLAS have presented reinterpretations of squark searches [126], although they only consider the decay of a leptoquark into 3rd generation quarks. We note that the ATLAS and CMS searches also consider leptoquark decays into down-type quarks and a neutrino (e.g $b \nu b \nu$ final states), but the corresponding couplings do not exist in our model and, therefore, we do not consider them here. 
Singly produced scalar leptoquarks have been searched in $e j, \mu j$, and $b \tau$ final states. The bounds on the leptoquark mass from single production depends on the coupling of the leptoquark to quarks and leptons. For unit couplings, $8 \mathrm{TeV}$ searches for single production of first and second generation scalar leptoquarks constrain the leptoquark mass to be above $\sim 1700 \mathrm{GeV}$ and $\sim 700 \mathrm{GeV}$, respectively [131], while the $13 \mathrm{TeV}$ search for third generation scalar leptoquarks constrains the mass to be above $740 \mathrm{GeV}$ [132]. In our benchmark models, the leptoquarks are mainly coupled to bottom or strange quarks. For this reason, the searches for singly produced leptoquarks are less sensitive to our benchmark models than the searches for pair produced leptoquarks. In the following, we will discuss in some details the bounds from searches of pair produced leptoquarks in all benchmarks.

For BM1 and BM2, the dominant non-zero couplings of $U_{1}$ are couplings involving tau leptons $\left(\lambda_{33}^{q}, \lambda_{23}^{q}\right)$ and the dominant decay modes are $U_{1} \rightarrow b \tau, s \tau, t \nu_{\tau}, c \nu_{\tau}$. At small values of $\lambda_{33}^{d}$ (see figure 2), the branching ratios of the $b \tau$ and $\tau \nu_{\tau}$ decay modes are similar in value $(\sim 0.25)$ and dominate over the $s \tau$ and $c \nu_{\tau}$ decays modes, which themselves have similar branching ratios $(\sim 0.18)$. For values of $\lambda_{33}^{d}$ near the border of the region allowed by $B_{s} \rightarrow \tau^{+} \tau^{-}$(see figure 2), the decay into $b \tau$ becomes the dominant decay mode with $\operatorname{BR}\left(U_{1} \rightarrow b \tau\right) \sim 0.4$.

The reinterpreted SUSY search for pair production of vector leptoquarks decaying to $t \nu$ [128] and the CMS search for leptoquarks decaying to $b \tau$ [127] are the most sensitive searches. We find that these searches yield a similar lower bound on the mass of $U_{1}$ at around $1.2 \mathrm{TeV}$ in the region of parameter space with small $\lambda_{33}^{d}$. The exact bound varies by at most $\sim 100 \mathrm{GeV}$ in the region allowed by $B_{s} \rightarrow \tau^{+} \tau^{-}$.

In BM3, $U_{1}$ couples dominantly to 2nd generation leptons and the main decay modes are $U_{1} \rightarrow b \mu, s \mu, t \nu_{\mu}, c \nu_{\mu}$, with the $b \mu$ and $t \nu_{\mu}$ decays modes being the dominant ones since $\lambda_{32}^{q} \gg \lambda_{22}^{q}, \mathrm{BR}\left(U_{1} \rightarrow t \nu_{\mu}\right) \sim \mathrm{BR}\left(U_{1} \rightarrow b \mu\right) \sim 0.5$. The most stringent LHC constraint on this benchmark comes from the search for pair produced leptoquarks in final states with two muons and two jets in [125]. ${ }^{3}$ This search leads to the bound $m_{U_{1}} \gtrsim 1.9 \mathrm{TeV}$. This bound is valid in the entire parameter space shown in the right panel figure 3 , since $\lambda_{32}^{d}$ is constrained to be very small, and therefore does not affect the leptoquark branching ratios.

Finally, in BM4, $U_{1}$ couples dominantly to 1st generation leptons and the main decay modes are $U_{1} \rightarrow$ be and $U_{1} \rightarrow t \nu_{e}$. In particular, at small values of $\lambda_{31}^{d}$ (see figure 4 ), the branching ratios of these decay modes are very similar in value $(\sim 0.5)$. At larger values of $\lambda_{31}^{d}$, the branching ratio into be becomes the dominant one, with $\operatorname{BR}\left(U_{1} \rightarrow b e\right) \sim 0.7$ at the border of the allowed region for $\lambda_{31}^{d}$, as shown in the right plot of figure 4 . The search for pair produced leptoquarks decaying in an electron and a jet in [124] provides the strongest constraint on the mass of $U_{1}$ and gives a lower bound of $\sim 1.8 \mathrm{TeV}$ at small values of $\lambda_{31}^{d}$. The exact bound varies by at most $\sim 100 \mathrm{GeV}$ in the region allowed by $B_{s} \rightarrow e^{+} e^{-}$.

\section{Conclusions}

In this study, we focused on the possible, and quite likely, existence of new sources of $\mathrm{CP}$ violation if the flavor anomalies in $b \rightarrow c$ and $b \rightarrow s$ decays are due to new physics,

\footnotetext{
${ }^{3}$ The search does not require any anti- $b$ tagging, and, therefore, we can simply apply it to our benchmark.
} 
specifically in the case where the new physics consists of a $U_{1}$ vector leptoquark. The underpinning of our study is that the $U_{1}$ vector leptoquark is one of the only (if not the only) new physics scenarios known to us that can provide a simultaneous explanation of the anomalies observed in lepton flavor universality ratios in $b \rightarrow c \ell \nu$ and $b \rightarrow s \ell \ell$ decays, $R_{D^{(*)}}$ and $R_{K^{(*)}}$. Since the couplings of the $U_{1}$ to quarks and leptons are generically CP violating, they are expected just as generically to produce potentially observable electric dipole moments (EDMs) in leptonic and hadronic systems. Here, we have first provided new, original, and complete formulae for the calculation of the relevant EDMs, and carried out a phenomenological study of a few benchmark cases of how EDMs can constrain the $U_{1}$ leptoquark interpretation of the anomalies.

We note that the expressions we provided are the most general expressions for dipole moments induced by vector leptoquarks at one loop level, accounting for the most generic set of leptoquark couplings, which can accomodate scenarios for which the leptoquark may be composite.

We explored the parameter space of the $U_{1}$ leptoquark in the vicinity of 4 benchmark points that explain the $R_{D^{(*)}}$ and $R_{K^{(*)}}$ anomalies (or a subset of them). We identified viable regions of parameter space where the existing discrepancies in the anomalous magnetic dipole moments of the electron $a_{e}$ and the muon $a_{\mu}$ can be explained in addition

to $R_{K}^{(*)}$. However, we concluded that a simultaneous explanation of all three classes of discrepancies $\left(R_{D^{(*)}}, R_{K^{(*)}}, a_{e, \mu}\right)$ is not possible.

We found that, in the presence of non-zero CP-violating phases in the leptoquark couplings, EDMs play an important role in probing the parameter space of the model. Existing bounds on the electron EDM already exclude large parts of parameter space with $\mathrm{CP}$ violating leptoquark couplings to electrons. The expected sensitivities to the neutron EDM can probe into motivated parameter space and probe imaginary parts of leptoquark couplings to taus and muons.

\section{Acknowledgments}

The research of WA is supported by the National Science Foundation under Grant No. PHY-1912719. WA acknowledges support by the Munich Institute for Astro- and Particle Physics (MIAPP) which is funded by the Deutsche Forschungsgemeinschaft (DFG, German Research Foundation) under Germany's Excellence Strategy — EXC-2094 — 390783311. The research of SG and DT is supported in part by the NSF CAREER grant PHY-1915852. SG would like to thank the Aspen Center for Physics under NSF grant PHY-1607611, where part of this work was performed. HHP and SP are partly supported by the U.S. Department of Energy grant number de-sc0010107. The work of DT is supported in part by the U.S. Department of Energy, Office of Science, Office of Workforce Development for Teachers and Scientists, Office of Science Graduate Student Research (SCGSR) program. The SCGSR program is administered by the Oak Ridge Institute for Science and Education for the DOE under contract number de-sc0014664. 
Open Access. This article is distributed under the terms of the Creative Commons Attribution License (CC-BY 4.0), which permits any use, distribution and reproduction in any medium, provided the original author(s) and source are credited.

\section{References}

[1] BABAR collaboration, Evidence for an excess of $\bar{B} \rightarrow D^{(*)} \tau^{-} \bar{\nu}_{\tau}$ decays, Phys. Rev. Lett. 109 (2012) 101802 [arXiv: 1205.5442] [INSPIRE].

[2] Belle collaboration, Measurement of the branching ratio of $\bar{B} \rightarrow D^{(*)} \tau^{-} \bar{\nu}_{\tau}$ relative to $\bar{B} \rightarrow D^{(*)} \ell^{-} \bar{\nu}_{\ell}$ decays with hadronic tagging at Belle, Phys. Rev. D 92 (2015) 072014 [arXiv: 1507.03233] [INSPIRE].

[3] Belle collaboration, Measurement of the $\tau$ lepton polarization and $R\left(D^{*}\right)$ in the decay $\bar{B} \rightarrow D^{*} \tau^{-} \bar{\nu}_{\tau}$, Phys. Rev. Lett. 118 (2017) 211801 [arXiv:1612.00529] [INSPIRE].

[4] Belle collaboration, Measurement of $\mathcal{R}(D)$ and $\mathcal{R}\left(D^{*}\right)$ with a semileptonic tagging method, Phys. Rev. Lett. 124 (2020) 161803 [arXiv:1910.05864] [InSPIRE].

[5] LHCb collaboration, Measurement of the ratio of branching fractions $\mathcal{B}\left(\bar{B}^{0} \rightarrow D^{*+} \tau^{-} \bar{\nu}_{\tau}\right) / \mathcal{B}\left(\bar{B}^{0} \rightarrow D^{*+} \mu^{-} \bar{\nu}_{\mu}\right)$, Phys. Rev. Lett. 115 (2015) 111803 [Erratum ibid. 115 (2015) 159901] [arXiv:1506.08614] [INSPIRE].

[6] LHCb collaboration, Measurement of the ratio of the $B^{0} \rightarrow D^{*-} \tau^{+} \nu_{\tau}$ and $B^{0} \rightarrow D^{*-} \mu^{+} \nu_{\mu}$ branching fractions using three-prong $\tau$-lepton decays, Phys. Rev. Lett. 120 (2018) 171802 [arXiv: 1708.08856] [INSPIRE].

[7] HFLAV collaboration, Averages of b-hadron, c-hadron and $\tau$-lepton properties as of 2018, arXiv:1909.12524 [INSPIRE].

[8] F.U. Bernlochner, Z. Ligeti, M. Papucci and D.J. Robinson, Combined analysis of semileptonic $B$ decays to $D$ and $D^{*}: R\left(D^{(*)}\right),\left|V_{c b}\right|$ and new physics, Phys. Rev. D 95 (2017) 115008 [arXiv: 1703.05330] [inSPIRE].

[9] D. Bigi, P. Gambino and S. Schacht, $R\left(D^{*}\right),\left|V_{c b}\right|$ and the heavy quark symmetry relations between form factors, JHEP 11 (2017) 061 [arXiv:1707.09509] [INSPIRE].

[10] S. Jaiswal, S. Nandi and S.K. Patra, Extraction of $\left|V_{c b}\right|$ from $B \rightarrow D^{(*)} \ell \nu_{\ell}$ and the standard model predictions of $R\left(D^{(*)}\right)$, JHEP 12 (2017) 060 [arXiv:1707.09977] [INSPIRE].

[11] LHCb collaboration, Search for lepton-universality violation in $B^{+} \rightarrow K^{+} \ell^{+} \ell^{-}$decays, Phys. Rev. Lett. 122 (2019) 191801 [arXiv:1903.09252] [inSPIRE].

[12] M. Bordone, G. Isidori and A. Pattori, On the standard model predictions for $R_{K}$ and $R_{K^{*}}$, Eur. Phys. J. C 76 (2016) 440 [arXiv: 1605.07633] [INSPIRE].

[13] LHCb collaboration, Test of lepton universality using $B^{+} \rightarrow K^{+} \ell^{+} \ell^{-}$decays, Phys. Rev. Lett. 113 (2014) 151601 [arXiv:1406.6482] [INSPIRE].

[14] LHCb collaboration, Test of lepton universality with $B^{0} \rightarrow K^{* 0} \ell^{+} \ell^{-}$decays, JHEP 08 (2017) 055 [arXiv: 1705. 05802] [inSPIRE].

[15] BeLLe collaboration, Test of lepton flavor universality in $B \rightarrow K^{*} \ell^{+} \ell^{-}$decays at Belle, arXiv: 1904.02440 [INSPIRE].

[16] BeLLe collaboration, Test of lepton flavor universality in $B \rightarrow K \ell^{+} \ell^{-}$decays, arXiv: 1908.01848 [INSPIRE]. 
[17] M. Algueró et al., Emerging patterns of new physics with and without lepton flavour universal contributions, Eur. Phys. J. C 79 (2019) 714 [arXiv: 1903.09578] [InSPIRE].

[18] A.K. Alok, A. Dighe, S. Gangal and D. Kumar, Continuing search for new physics in $b \rightarrow s \mu \mu$ decays: two operators at a time, JHEP 06 (2019) 089 [arXiv:1903.09617] [INSPIRE].

[19] M. Ciuchini et al., New Physics in $b \rightarrow s \ell^{+} \ell^{-}$confronts new data on Lepton Universality, Eur. Phys. J. C 79 (2019) 719 [arXiv:1903.09632] [INSPIRE].

[20] A. Datta, J. Kumar and D. London, The $B$ anomalies and new physics in $b \rightarrow s e^{+} e^{-}$, Phys. Lett. B 797 (2019) 134858 [arXiv:1903.10086].

[21] J. Aebischer et al., B-decay discrepancies after Moriond 2019, Eur. Phys. J. C 80 (2020) 252 [arXiv: 1903.10434] [INSPIRE].

[22] K. Kowalska, D. Kumar and E.M. Sessolo, Implications for new physics in $b \rightarrow s \mu \mu$ transitions after recent measurements by Belle and LHCb, Eur. Phys. J. C 79 (2019) 840 [arXiv: 1903.10932] [INSPIRE].

[23] A. Arbey et al., Update on the $b \rightarrow s$ anomalies, Phys. Rev. D 100 (2019) 015045 [arXiv: 1904.08399] [INSPIRE].

[24] Planck collaboration, Planck 2015 results. XIII. Cosmological parameters, Astron. Astrophys. 594 (2016) A13 [arXiv:1502.01589] [INSPIRE].

[25] A.D. Sakharov, Violation of CP Invariance, $C$ asymmetry and baryon asymmetry of the universe, Pisma Zh. Eksp. Teor. Fiz. 5 (1967) 32 [INSPIRE].

[26] R. Alonso, B. Grinstein and J. Martin Camalich, Lepton universality violation and lepton flavor conservation in B-meson decays, JHEP 10 (2015) 184 [arXiv: 1505.05164] [INSPIRE].

[27] B. Bhattacharya et al., Simultaneous explanation of the $R_{K}$ and $R_{D^{(*)}}$ puzzles: a model analysis, JHEP 01 (2017) 015 [arXiv: 1609.09078] [inSPIRE].

[28] D. Buttazzo, A. Greljo, G. Isidori and D. Marzocca, B-physics anomalies: a guide to combined explanations, JHEP 11 (2017) 044 [arXiv: 1706. 07808] [INSPIRE].

[29] L. Calibbi, A. Crivellin and T. Li, Model of vector leptoquarks in view of the B-physics anomalies, Phys. Rev. D 98 (2018) 115002 [arXiv:1709.00692] [INSPIRE].

[30] A. Crivellin, C. Greub, D. Müller and F. Saturnino, Importance of loop effects in explaining the accumulated evidence for new physics in B decays with a vector leptoquark, Phys. Rev. Lett. 122 (2019) 011805 [arXiv: 1807.02068] [INSPIRE].

[31] A. Angelescu, D. Bečirević, D.A. Faroughy and O. Sumensari, Closing the window on single leptoquark solutions to the B-physics anomalies, JHEP 10 (2018) 183 [arXiv:1808.08179] [INSPIRE].

[32] C. Cornella, J. Fuentes-Martin and G. Isidori, Revisiting the vector leptoquark explanation of the B-physics anomalies, JHEP 07 (2019) 168 [arXiv:1903.11517] [INSPIRE].

[33] G. Panico, A. Pomarol and M. Riembau, EFT approach to the electron electric dipole moment at the two-loop level, JHEP 04 (2019) 090 [arXiv: 1810.09413] [INSPIRE].

[34] P. Herczeg, CP violating electron-nucleon interactions from leptoquark exchange, Phys. Rev. D 68 (2003) 116004 [Erratum ibid. D 69 (2004) 039901] [INSPIRE]. 
[35] N. Yamanaka et al., Probing exotic phenomena at the interface of nuclear and particle physics with the electric dipole moments of diamagnetic atoms: A unique window to hadronic and semi-leptonic CP-violation, Eur. Phys. J. A 53 (2017) 54 [arXiv: 1703.01570] [INSPIRE].

[36] C. Bobeth and A.J. Buras, Leptoquarks meet $\varepsilon^{\prime} / \varepsilon$ and rare Kaon processes, JHEP 02 (2018) 101 [arXiv: 1712.01295] [INSPIRE].

[37] K. Fuyuto, M. Ramsey-Musolf and T. Shen, Electric dipole moments from CP-violating scalar leptoquark interactions, Phys. Lett. B 788 (2019) 52 [arXiv:1804.01137] [InSPIRE].

[38] K. Yanase, N. Yoshinaga, K. Higashiyama and N. Yamanaka, Electric dipole moment of ${ }^{199} \mathrm{Hg}$ atom from P, CP-odd electron-nucleon interaction, Phys. Rev. D 99 (2019) 075021 [arXiv: 1805.00419] [INSPIRE].

[39] W. Dekens, J. de Vries, M. Jung and K.K. Vos, The phenomenology of electric dipole moments in models of scalar leptoquarks, JHEP 01 (2019) 069 [arXiv:1809.09114] [INSPIRE].

[40] A. Crivellin and F. Saturnino, Correlating tauonic B decays with the neutron electric dipole moment via a scalar leptoquark, Phys. Rev. D 100 (2019) 115014 [arXiv:1905.08257] [INSPIRE].

[41] M. Bordone, C. Cornella, J. Fuentes-Martin and G. Isidori, A three-site gauge model for flavor hierarchies and flavor anomalies, Phys. Lett. B 779 (2018) 317 [arXiv:1712.01368] [INSPIRE].

[42] M. Bordone, C. Cornella, J. Fuentes-Martín and G. Isidori, Low-energy signatures of the $P S^{3}$ model: from B-physics anomalies to LFV, JHEP 10 (2018) 148 [arXiv:1805.09328] [INSPIRE].

[43] N. Assad, B. Fornal and B. Grinstein, Baryon number and lepton universality violation in leptoquark and diquark models, Phys. Lett. B 777 (2018) 324 [arXiv:1708.06350] [INSPIRE].

[44] L. Di Luzio, A. Greljo and M. Nardecchia, Gauge leptoquark as the origin of B-physics anomalies, Phys. Rev. D 96 (2017) 115011 [arXiv:1708.08450] [INSPIRE].

[45] R. Barbieri and A. Tesi, B-decay anomalies in Pati-Salam SU(4), Eur. Phys. J. C 78 (2018) 193 [arXiv: 1712.06844] [INSPIRE].

[46] M. Blanke and A. Crivellin, B meson anomalies in a Pati-Salam model within the Randall-Sundrum background, Phys. Rev. Lett. 121 (2018) 011801 [arXiv:1801.07256] [INSPIRE].

[47] A. Greljo and B.A. Stefanek, Third family quark-lepton unification at the TeV scale, Phys. Lett. B 782 (2018) 131 [arXiv: 1802.04274] [INSPIRE].

[48] J. Heeck and D. Teresi, Pati-Salam explanations of the B-meson anomalies, JHEP 12 (2018) 103 [arXiv: 1808.07492] [InSPIRE].

[49] S. Balaji, R. Foot and M.A. Schmidt, Chiral SU(4) explanation of the $b \rightarrow s$ anomalies, Phys. Rev. D 99 (2019) 015029 [arXiv:1809.07562] [INSPIRE].

[50] B. Fornal, S.A. Gadam and B. Grinstein, Left-Right SU(4) vector leptoquark model for flavor anomalies, Phys. Rev. D 99 (2019) 055025 [arXiv: 1812.01603] [INSPIRE]. 
[51] I. Doršner et al., Physics of leptoquarks in precision experiments and at particle colliders, Phys. Rept. 641 (2016) 1 [arXiv: 1603.04993] [INSPIRE].

[52] D.J. Robinson, B. Shakya and J. Zupan, Right-handed neutrinos and $R\left(D^{(*)}\right)$, JHEP 02 (2019) 119 [arXiv: 1807.04753] [INSPIRE].

[53] F. Sala and D.M. Straub, A new light particle in B decays?, Phys. Lett. B 774 (2017) 205 [arXiv: 1704.06188] [INSPIRE].

[54] D. Ghosh, Explaining the $R_{K}$ and $R_{K^{*}}$ anomalies, Eur. Phys. J. C 77 (2017) 694 [arXiv: 1704.06240] [INSPIRE].

[55] A. Datta, J. Kumar, J. Liao and D. Marfatia, New light mediators for the $R_{K}$ and $R_{K^{*}}$ puzzles, Phys. Rev. D 97 (2018) 115038 [arXiv:1705.08423] [INSPIRE].

[56] W. Altmannshofer et al., Light resonances and the low- $q^{2}$ bin of $R_{K^{*}}, J H E P 03$ (2018) 188 [arXiv: 1711.07494] [INSPIRE].

[57] BABAR collaboration, Improved limits on lepton flavor violating $\tau$ decays to $l \phi, l \rho, l K^{*}$ and lK $\bar{K}^{*}$, Phys. Rev. Lett. 103 (2009) 021801 [arXiv:0904.0339] [INSPIRE].

[58] BeLLE collaboration, Search for lepton-flavor-violating $\tau$ decays into a lepton and a vector meson, Phys. Lett. B 699 (2011) 251 [arXiv:1101.0755] [INSPIRE].

[59] BABAr collaboration, A search for the decay modes $B^{ \pm} \rightarrow h^{ \pm} \tau^{ \pm} l$, Phys. Rev. D 86 (2012) 012004 [arXiv: 1204.2852] [INSPIRE].

[60] A. Greljo and D. Marzocca, High-p $p_{T}$ dilepton tails and flavor physics, Eur. Phys. J. C 77 (2017) 548 [arXiv: 1704.09015] [INSPIRE].

[61] D.A. Faroughy, A. Greljo and J.F. Kamenik, Confronting lepton flavor universality violation in B decays with high-p $p_{T}$ tau lepton searches at LHC, Phys. Lett. B 764 (2017) 126 [arXiv: 1609.07138] [INSPIRE].

[62] ALEPH, DELPHI, L3, OPAL, LEP EleCtroweAK collaboration, Electroweak measurements in electron-positron collisions at W-boson-pair energies at LEP, Phys. Rept. 532 (2013) 119 [arXiv:1302.3415] [INSPIRE].

[63] F.S. Queiroz and W. Shepherd, New physics contributions to the muon anomalous magnetic moment: a numerical code, Phys. Rev. D 89 (2014) 095024 [arXiv:1403.2309] [InSPIRE].

[64] K. Kowalska, E.M. Sessolo and Y. Yamamoto, Constraints on charmphilic solutions to the muon g- 2 with leptoquarks, Phys. Rev. D 99 (2019) 055007 [arXiv:1812.06851] [INSPIRE].

[65] CMS collaboration, Search for anomalous top chromomagnetic dipole moments from angular distributions in $t \bar{t}$ dileptonic events at $\sqrt{s}=7 \mathrm{TeV}$ with the CMS detector, CMS-PAS-TOP-14-005 (2014).

[66] J. Engel, M.J. Ramsey-Musolf and U. van Kolck, Electric dipole moments of nucleons, nuclei and atoms: the standard model and beyond, Prog. Part. Nucl. Phys. 71 (2013) 21 [arXiv:1303.2371] [INSPIRE].

[67] T. Bhattacharya et al., Neutron electric dipole moment and tensor charges from lattice QCD, Phys. Rev. Lett. 115 (2015) 212002 [arXiv:1506. 04196] [INSPIRE].

[68] T. Bhattacharya et al., Axial, scalar and tensor charges of the nucleon from $2+1+1$-flavor lattice QCD, Phys. Rev. D 94 (2016) 054508 [arXiv:1606.07049] [INSPIRE]. 
[69] D. Chang, W.-Y. Keung, C.S. Li and T.C. Yuan, QCD corrections to CP violation from color electric dipole moment of b quark, Phys. Lett. B 241 (1990) 589 [INSPIRE].

[70] G. Boyd, A.K. Gupta, S.P. Trivedi and M.B. Wise, Effective Hamiltonian for the electric dipole moment of the neutron, Phys. Lett. B 241 (1990) 584 [INSPIRE].

[71] M. Dine and W. Fischler, Constraints on new physics from Weinberg's analysis of the neutron electric dipole moment, Phys. Lett. B 242 (1990) 239 [INSPIRE].

[72] D.A. Demir, M. Pospelov and A. Ritz, Hadronic EDMs, the Weinberg operator and light gluinos, Phys. Rev. D 67 (2003) 015007 [hep-ph/0208257] [INSPIRE].

[73] U. Haisch and A. Hala, Sum rules for CP-violating operators of Weinberg type, JHEP 11 (2019) 154 [arXiv: 1909.08955] [InSPIRE].

[74] D. Hanneke, S. Fogwell and G. Gabrielse, New measurement of the electron magnetic moment and the fine structure constant, Phys. Rev. Lett. 100 (2008) 120801 [arXiv:0801.1134] [INSPIRE].

[75] Muon G-2 collaboration, Final report of the muon E821 anomalous magnetic moment measurement at BNL, Phys. Rev. D 73 (2006) 072003 [hep-ex/0602035] [INSPIRE].

[76] A. Keshavarzi, D. Nomura and T. Teubner, $g-2$ of charged leptons, $\alpha\left(M_{Z}^{2}\right)$ and the hyperfine splitting of muonium, Phys. Rev. D 101 (2020) 014029 [arXiv:1911.00367] [INSPIRE].

[77] H. Davoudiasl and W.J. Marciano, Tale of two anomalies, Phys. Rev. D 98 (2018) 075011 [arXiv: 1806.10252] [INSPIRE].

[78] M. Davier, A. Hoecker, B. Malaescu and Z. Zhang, A new evaluation of the hadronic vacuum polarisation contributions to the muon anomalous magnetic moment and to $\alpha\left(m_{Z}^{2}\right)$, Eur. Phys. J. C 80 (2020) 241 [arXiv: 1908.00921] [InSPIRE].

[79] A. Crivellin, M. Hoferichter and P. Schmidt-Wellenburg, Combined explanations of $(g-2)_{\mu, e}$ and implications for a large muon EDM, Phys. Rev. D 98 (2018) 113002 [arXiv: 1807.11484] [INSPIRE].

[80] F. Jegerlehner, Muon g- 2 theory: the hadronic part, EPJ Web Conf. 166 (2018) 00022 [arXiv: 1705. 00263] [INSPIRE].

[81] R.H. Parker, C. Yu, W. Zhong, B. Estey and H. Müller, Measurement of the fine-structure constant as a test of the Standard Model, Science 360 (2018) 191 [arXiv:1812.04130] [INSPIRE].

[82] Muon G-2 collaboration, Muon $(g-2)$ technical design report, arXiv:1501.06858 [INSPIRE].

[83] T. Blum et al., Connected and leading disconnected hadronic light-by-light contribution to the muon anomalous magnetic moment with a physical pion mass, Phys. Rev. Lett. 118 (2017) 022005 [arXiv: 1610.04603] [INSPIRE].

[84] RBC, UKQCD collaboration, Calculation of the hadronic vacuum polarization contribution to the muon anomalous magnetic moment, Phys. Rev. Lett. 121 (2018) 022003 [arXiv: 1801.07224] [INSPIRE].

[85] Fermilab Lattice, LATTICE-HPQCD, MiLC collaboration, Hadronic-vacuum-polarization contribution to the muon's anomalous magnetic moment from four-flavor lattice QCD, Phys. Rev. D 101 (2020) 034512 [arXiv: 1902.04223] [INSPIRE]. 
[86] PACS collaboration, Hadronic vacuum polarization contribution to the muon $g-2$ with $2+1$ flavor lattice $Q C D$ on a larger than $(10 \mathrm{fm})^{4}$ lattice at the physical point, Phys. Rev. D 100 (2019) 034517 [arXiv:1902.00885] [INSPIRE].

[87] A. Gérardin et al., The leading hadronic contribution to $(g-2)_{\mu}$ from lattice $Q C D$ with $N_{\mathrm{f}}=2+1$ flavours of $O(a)$ improved Wilson quarks, Phys. Rev. D 100 (2019) 014510 [arXiv: 1904.03120] [INSPIRE].

[88] T. Blum et al., The hadronic light-by-light scattering contribution to the muon anomalous magnetic moment from lattice QCD, Phys. Rev. Lett. 124 (2020) 132002 [arXiv: 1911.08123] [INSPIRE].

[89] G. Colangelo, M. Hoferichter, M. Procura and P. Stoffer, Dispersion relation for hadronic light-by-light scattering: theoretical foundations, JHEP 09 (2015) 074 [arXiv:1506.01386] [INSPIRE].

[90] G. Colangelo, M. Hoferichter, M. Procura and P. Stoffer, Rescattering effects in the hadronic-light-by-light contribution to the anomalous magnetic moment of the muon, Phys. Rev. Lett. 118 (2017) 232001 [arXiv:1701.06554] [INSPIRE].

[91] M. Hoferichter, B.-L. Hoid, B. Kubis, S. Leupold and S.P. Schneider, Pion-pole contribution to hadronic light-by-light scattering in the anomalous magnetic moment of the muon, Phys. Rev. Lett. 121 (2018) 112002 [arXiv:1805.01471] [INSPIRE].

[92] G. Colangelo, M. Hoferichter and P. Stoffer, Two-pion contribution to hadronic vacuum polarization, JHEP 02 (2019) 006 [arXiv:1810.00007] [INSPIRE].

[93] M. Hoferichter, B.-L. Hoid and B. Kubis, Three-pion contribution to hadronic vacuum polarization, JHEP 08 (2019) 137 [arXiv:1907.01556] [INSPIRE].

[94] G. Gabrielse, S.E. Fayer, T.G. Myers and X. Fan, Towards an improved test of the standard model's most precise prediction, Atoms 7 (2019) 45 [arXiv:1904.06174] [INSPIRE].

[95] DELPHI collaboration, Study of tau-pair production in photon-photon collisions at LEP and limits on the anomalous electromagnetic moments of the tau lepton, Eur. Phys. J. C 35 (2004) 159 [hep-ex/0406010] [INSPIRE].

[96] A. Pich, Precision Tau Physics, Prog. Part. Nucl. Phys. 75 (2014) 41 [arXiv:1310.7922] [INSPIRE].

[97] ACME collaboration, Improved limit on the electric dipole moment of the electron, Nature 562 (2018) 355 [INSPIRE].

[98] Muon (G-2) collaboration, An improved limit on the muon electric dipole moment, Phys. Rev. D 80 (2009) 052008 [arXiv: 0811.1207] [InSPIRE].

[99] Belle collaboration, Search for the electric dipole moment of the tau lepton, Phys. Lett. B 551 (2003) 16 [hep-ex/0210066] [INSPIRE].

[100] A. Adelmann, K. Kirch, C.J.G. Onderwater and T. Schietinger, Compact storage ring to search for the muon electric dipole moment, J. Phys. G 37 (2010) 085001 [InSPIRE].

[101] BeLLE-II collaboration, The Belle II physics book, PTEP 2019 (2019) 123C01 [Erratum ibid. 2020 (2020) 029201] [arXiv:1808.10567] [INSPIRE].

[102] NEDM collaboration, Measurement of the permanent electric dipole moment of the neutron, Phys. Rev. Lett. 124 (2020) 081803 [arXiv: 2001.11966] [INSPIRE].

[103] R.K. Ellis et al., Physics briefing book, arXiv:1910.11775 [INSPIRE]. 
[104] M. Pospelov and A. Ritz, CKM benchmarks for electron electric dipole moment experiments, Phys. Rev. D 89 (2014) 056006 [arXiv:1311.5537] [INSPIRE].

[105] C. Smith and S. Touati, Electric dipole moments with and beyond flavor invariants, Nucl. Phys. B 924 (2017) 417 [arXiv: 1707.06805] [INSPIRE].

[106] S. Dar, The neutron EDM in the SM: a review, hep-ph/0008248 [INSPIRE].

[107] X.-Q. Li, Y.-D. Yang and X. Zhang, Revisiting the one leptoquark solution to the $R\left(D^{(*)}\right)$ anomalies and its phenomenological implications, JHEP 08 (2016) 054 [arXiv: 1605.09308] [INSPIRE].

[108] R. Alonso, B. Grinstein and J. Martin Camalich, Lifetime of $B_{c}^{-}$constrains explanations for anomalies in $B \rightarrow D^{(*)} \tau \nu$, Phys. Rev. Lett. 118 (2017) 081802 [arXiv:1611.06676] [INSPIRE].

[109] W. Altmannshofer, C. Niehoff and D.M. Straub, $B_{s} \rightarrow \mu^{+} \mu^{-}$as current and future probe of new physics, JHEP 05 (2017) 076 [arXiv:1702.05498] [INSPIRE].

[110] W. Altmannshofer et al., Symmetries and asymmetries of $B \rightarrow K^{*} \mu^{+} \mu^{-}$decays in the standard model and beyond, JHEP 01 (2009) 019 [arXiv:0811.1214] [INSPIRE].

[111] Particle Data Group collaboration, Review of particle physics, Phys. Rev. D 98 (2018) 030001 [INSPIRE].

[112] CKMfitter Group collaboration, CP violation and the CKM matrix: Assessing the impact of the asymmetric B factories, Eur. Phys. J. C 41 (2005) 1 [hep-ph/0406184] [INSPIRE].

[113] C. McNeile et al., Heavy meson masses and decay constants from relativistic heavy quarks in full lattice QCD, Phys. Rev. D 86 (2012) 074503 [arXiv:1207.0994] [inSPIRE].

[114] LHCb collaboration, Search for the decays $B_{s}^{0} \rightarrow \tau^{+} \tau^{-}$and $B^{0} \rightarrow \tau^{+} \tau^{-}$, Phys. Rev. Lett. 118 (2017) 251802 [arXiv: 1703.02508] [INSPIRE].

[115] C. Bobeth et al., $B_{s, d} \rightarrow l^{+} l^{-}$in the standard model with reduced theoretical uncertainty, Phys. Rev. Lett. 112 (2014) 101801 [arXiv:1311.0903] [InSPIRE].

[116] J. Albrecht et al., Future prospects for exploring present day anomalies in flavour physics measurements with Belle II and LHCb, arXiv:1709.10308 [INSPIRE].

[117] CMS collaboration, Measurement of the $B_{s}^{0} \rightarrow \mu^{+} \mu^{-}$branching fraction and search for $B^{0} \rightarrow \mu^{+} \mu^{-}$with the CMS experiment, Phys. Rev. Lett. 111 (2013) 101804 [arXiv: 1307.5025] [INSPIRE].

[118] CMS, LHCB collaboration, Observation of the rare $B_{s}^{0} \rightarrow \mu^{+} \mu^{-}$decay from the combined analysis of CMS and LHCb data, Nature 522 (2015) 68 [arXiv:1411.4413] [INSPIRE].

[119] LHCb collaboration, Measurement of the $B_{s}^{0} \rightarrow \mu^{+} \mu^{-}$branching fraction and effective lifetime and search for $B^{0} \rightarrow \mu^{+} \mu^{-}$decays, Phys. Rev. Lett. 118 (2017) 191801 [arXiv: 1703.05747] [INSPIRE].

[120] ATLAS collaboration, Study of the rare decays of $B_{s}^{0}$ and $B^{0}$ mesons into muon pairs using data collected during 2015 and 2016 with the ATLAS detector, JHEP 04 (2019) 098 [arXiv: 1812.03017] [INSPIRE].

[121] M. Beneke, C. Bobeth and R. Szafron, Enhanced electromagnetic correction to the rare B-meson decay $B_{s, d} \rightarrow \mu^{+} \mu^{-}$, Phys. Rev. Lett. 120 (2018) 011801 [arXiv:1708.09152] [INSPIRE]. 
[122] CDF collaboration, Search for the decays $B_{s}^{0} \rightarrow e^{+} \mu^{-}$and $B_{s}^{0} \rightarrow e^{+} e^{-}$in CDF Run II, Phys. Rev. Lett. 102 (2009) 201801 [arXiv:0901.3803] [InSPIRE].

[123] ATLAS collaboration, Searches for scalar leptoquarks and differential cross-section measurements in dilepton-dijet events in proton-proton collisions at a centre-of-mass energy of $\sqrt{s}=13 \mathrm{TeV}$ with the ATLAS experiment, Eur. Phys. J. C 79 (2019) 733 [arXiv: 1902.00377] [INSPIRE].

[124] CMS collaboration, Search for pair production of first-generation scalar leptoquarks at $\sqrt{s}=13$ TeV, Phys. Rev. D 99 (2019) 052002 [arXiv: 1811.01197] [INSPIRE].

[125] CMS collaboration, Search for pair production of second-generation leptoquarks at $\sqrt{s}=13$ TeV, Phys. Rev. D 99 (2019) 032014 [arXiv:1808.05082] [INSPIRE].

[126] ATLAS collaboration, Searches for third-generation scalar leptoquarks in $\sqrt{s}=13 \mathrm{TeV} \mathrm{pp}$ collisions with the ATLAS detector, JHEP 06 (2019) 144 [arXiv:1902.08103] [INSPIRE].

[127] CMS collaboration, Search for heavy neutrinos and third-generation leptoquarks in hadronic states of two $\tau$ leptons and two jets in proton-proton collisions at $\sqrt{s}=13$ TeV, JHEP 03 (2019) 170 [arXiv: 1811.00806] [INSPIRE].

[128] CMS collaboration, Constraints on models of scalar and vector leptoquarks decaying to a quark and a neutrino at $\sqrt{s}=13 \mathrm{TeV}$, Phys. Rev. D 98 (2018) 032005 [arXiv:1805.10228] [INSPIRE].

[129] B. Diaz, M. Schmaltz and Y.-M. Zhong, The leptoquark Hunter's guide: pair production, JHEP 10 (2017) 097 [arXiv: 1706. 05033] [INSPIRE].

[130] I. Doršner and A. Greljo, Leptoquark toolbox for precision collider studies, JHEP 05 (2018) 126 [arXiv: 1801.07641] [INSPIRE].

[131] CMS collaboration, Search for single production of scalar leptoquarks in proton-proton collisions at $\sqrt{s}=8 \mathrm{TeV}$, Phys. Rev. D 93 (2016) 032005 [Erratum ibid. D 95 (2017) 039906] [arXiv: 1509.03750] [INSPIRE].

[132] CMS collaboration, Search for a singly produced third-generation scalar leptoquark decaying to a $\tau$ lepton and a bottom quark in proton-proton collisions at $\sqrt{s}=13 \mathrm{TeV}, \mathrm{JHEP} \mathbf{0 7}$ (2018) 115 [arXiv: 1806.03472] [INSPIRE]. 